\title{
Orbit insertion strategy of Hayabusa2's rover with large release uncertainty around the asteroid Ryugu
}

\author{
Yusuke $\mathrm{Oki}^{1}(\bowtie)$, Kent Yoshikawa ${ }^{2}$, Hiroshi Takeuchi ${ }^{2}$, Shota Kikuchi ${ }^{2}$, Hitosi Ikeda ${ }^{2}$, Daniel \\ J. Scheeres ${ }^{3}$, Jay W. McMahon ${ }^{3}$, Junichiro Kawaguchi ${ }^{2}$, Yuto Takei ${ }^{2}$, Yuya Mimasu ${ }^{2}$, Naoko \\ Ogawa $^{2}$, Go Ono ${ }^{2}$, Fuyuto Terui ${ }^{2}$, Manabu Yamada ${ }^{4}$, Toru Kouyama ${ }^{5}$, Shingo Kameda ${ }^{6}$, Kazuya \\ Yoshida $^{7}$, Kenji Nagaoka ${ }^{8}$, Tetsuo Yoshimitsu ${ }^{2}$, Takanao Saiki ${ }^{2}$, and Yuichi Tsuda ${ }^{2}$ \\ 1. Japan Aerospace Exploration Agency, Tsukuba 305-8505, Japan \\ 2. Japan Aerospace Exploration Agency, Sagamihara 252-5210, Japan \\ 3. University of Colorado, Boulder, CO 80309, USA \\ 4. Planetary Exploration Research Center, Chiba Institute of Technology, Narashino 275-0016, Japan \\ 5. National Institute of Advanced Industrial Science and Technology, Koto-ku 135-0064, Japan \\ 6. Rikkyo University, Toshima-ku 171-8501, Japan \\ 7. Department of Aerospace Engineering, Tohoku University, Sendai 980-8579, Japan \\ 8. Kyushu Institute of Technology, Kitakyusyu 804-8550, Japan
}

\begin{abstract}
This paper describes the orbit design of the deployable payload Rover 2 of MINERVA-II, installed on the Hayabusa2 spacecraft. Because Rover 2 did not have surface exploration capabilities, the operation team decided to experiment with a new strategy for its deployment to the surface. The rover was ejected at a high altitude and made a semi-hard landing on the surface of the asteroid Ryugu after several orbits. Based on the orbital analysis around Ryugu, the expected collision speed was tolerable for the rover to function post-impact. Because the rover could not control its position, its motion was entirely governed by the initial conditions. Thus, the largest challenge was to insert the rover into a stable orbit (despite its large release uncertainty), and avoid its escape from Ryugu due to an environment strongly perturbed by solar radiation pressure and gravitational irregularities. This study investigates the solution space of the orbit around Ryugu and evaluates the orbit's robustness by utilizing Monte Carlo simulations to determine the orbit insertion policy. Upon analyzing the flight data of the rover operation, we verified that the rover orbited Ryugu for more than one period and established the possibility of a novel method for estimating the gravity of an asteroid.
\end{abstract}

\section{KEYWORDS}

solar radiation pressure augmented elliptic Hill three-

body problem

gravitational irregularity

\section{Research Article}

Received: 21 Januany 2020

Accepted: 7 April 2020

C) The Author(s) 2020,

Corrected Publication

January 2021

\section{Introduction}

The exploration of asteroids is receiving much attention among space agencies around the world, given the potential for elucidating how the solar system was created and evolved. Japan Aerospace Exploration Agency (JAXA)'s deep space exploration probe Hayabusa2 was launched in December 2014 and rendezvoused with its target asteroid, Ryugu, in June 2018. After Hayabusa2 conducted a global mapping and estimated the gravity model of Ryugu, it successfully made two touchdowns and sample collections on the surface of Ryugu, in February and July, 2019. Hayabusa2 remained in proximity to Ryugu, conducting operations related to touchdown and the rover release operation. This paper describes the orbital analyses with respect to the planning and results of one rover operation.

Hayabusa2 has several rovers, one of which is called "Rover 2 of MINERVA II-2" [1,2]. Figure 1 shows the appearance of the MINERVA II-2 system. We 


\section{Nomenclature}

\begin{tabular}{|c|c|}
\hline $\boldsymbol{r}=[x, y, z]$ & position vector and its elements in cartesian coordinates \\
\hline $\boldsymbol{a}_{\mathrm{G}}=\left[a_{\mathrm{G} x}, a_{\mathrm{G} y}, a_{\mathrm{G} z}\right]$ & normalized gravitational acceleration \\
\hline$e_{\mathrm{r}}$ & eccentricity of asteroid's orbit \\
\hline$a_{\mathrm{r}}$ & semi-major axis of asteroid's orbit $(\mathrm{m})$ \\
\hline$f_{\mathrm{r}}$ & true anomaly of asteroid's orbit (rad) \\
\hline$U$ & normalized gravity potential associated with asteroid harmonics \\
\hline$d$ & distance between the Sun and asteroid \\
\hline LU & distance unit \\
\hline TU & time unit \\
\hline$\beta$ & normalized acceleration by solar radiation pressure \\
\hline$a_{\mathrm{SRP}}$ & unnormalized acceleration by solar radiation pressure $\left(\mathrm{m} / \mathrm{s}^{2}\right)$ \\
\hline$\gamma$ & reflectivity \\
\hline$p_{0}$ & solar flux constant $\left(\mathrm{kg} \cdot \mathrm{m} / \mathrm{s}^{2}\right)$ \\
\hline$m$ & mass of the rover $(\mathrm{kg})$ \\
\hline$A$ & projected area of the rover $\left(\mathrm{m}^{2}\right)$ \\
\hline$\mu_{\mathrm{S}}$ & gravitational parameter of the Sun $\left(\mathrm{m}^{3} / \mathrm{s}^{2}\right)$ \\
\hline$\mu$ & gravitational parameter of the asteroid $\left(\mathrm{m}^{3} / \mathrm{s}^{2}\right)$ \\
\hline$P$ & legendre polynomial \\
\hline$l, m$ & order and degree of harmonics taken into account \\
\hline$C_{l m}, S_{l m}$ & stocks coefficients \\
\hline$\phi$ & latitude in asteroid fixed frame (rad) \\
\hline$\lambda$ & longitude (rad) \\
\hline$n$ & mean motion $(\mathrm{rad} / \mathrm{s})$ \\
\hline$C_{\mathrm{J}}$ & Jacobi integral $\left(\mathrm{m}^{2} / \mathrm{s}^{2}\right)$ \\
\hline$v_{\mathrm{c}}$ & critical velocity $(\mathrm{m} / \mathrm{s})$ \\
\hline$v_{\mathrm{o}}$ & circular orbit velocity in two-body problem $(\mathrm{m} / \mathrm{s})$ \\
\hline$v_{\mathrm{m}}$ & velocity margin $(\mathrm{m} / \mathrm{s})$ \\
\hline$a$ & semi-major axis of spacecraft's orbit $(\mathrm{m})$ \\
\hline$e$ & eccentricity of spacecraft's orbit \\
\hline$I$ & inclination of spacecraft's orbit \\
\hline$W$ & longitude of the ascending node of spacecraft's orbit \\
\hline$w$ & augment of periapsis of spacecraft's orbit \\
\hline$f$ & true anomaly of spacecraft's orbit \\
\hline
\end{tabular}

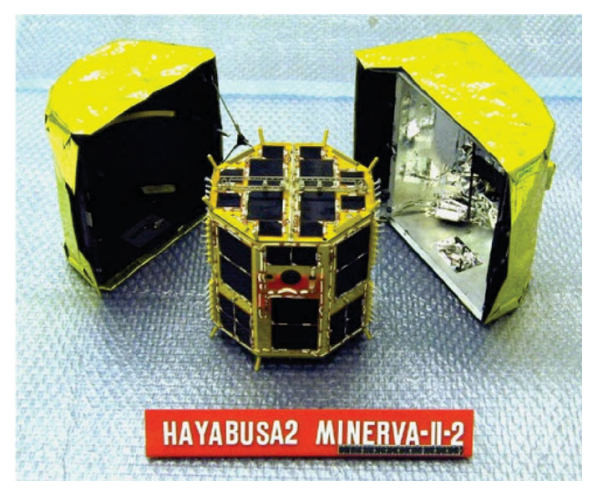

Fig. 1 MINERVA II-2 system.

determined that Rover 2 encountered some trouble after Hayabusa2 was launched. Its onboard computer did not function correctly due to hardware and software faults. Although it could emit radio waves when its communication equipment was powered on, if the rover was released to the surface of Ryugu, according to nominal operation, it would not be able to obtain surface data and send it to the mother spacecraft. Therefore, we planned to insert the rover into orbit and make a semihard landing on the surface after several orbits. Based on a basic orbital analysis around Ryugu, if Rover 2 was deployed at an altitude of $1.0 \mathrm{~km}$ above the surface, its impact with Ryugu would be slower than $25 \mathrm{~cm} / \mathrm{s}$. This expected collision speed was tolerable for the rover to function after impact.

The greatest scientific significance of orbiting the rover around Ryugu was its contribution to the construction of Ryugu's gravity model via determining the rover's orbit; this was achieved using an optical camera mounted on the mother spacecraft. By using the rover's orbit to accurately estimate the gravity field, we established the possibility of a novel method for estimating the gravity of asteroids via the orbit of a small probe. 
The rover must avoid escaping from Ryugu and ultimately land on the surface. However, as the design of Rover 2 did not account for orbit around Ryugu, inserting it into orbit entailed some difficulties. Primarily, the rover had a large release velocity uncertainty. The rover's release velocity was estimated to be between 5.4 and $25.4 \mathrm{~cm} / \mathrm{s}$. Moreover, there were guidance and control errors regarding the mother spacecraft's position and velocity. Hence, to overcome these difficulties, this study investigated stable orbits around Ryugu, notwithstanding large errors regarding the initial conditions.

This study first defines the coordinate systems used for both orbit design and spacecraft operation. It then demonstrates the equations of motion, including Ryugu's gravity and shape model. The main part of this paper describes how the rover's insertion policy was determined based on both an analytical perspective, such as the Jacobi integral, and the numerical solution space. Some trade-offs, such as whether a polar or equatorial orbit were more appropriate, are considered from the standpoints of stability and operational safety. Finally, this paper describes the results of the actual Rover 2 operation. The actual rover orbit was estimated based on flight data from several images taken by Hayabusa2's optical camera. We analyzed the rover's operation flight data, verified that the rover rotated around Ryugu for more than one period, and established the possibility of a novel method for estimating the gravity of an asteroid.

\section{Coordinate system}

This section defines the three main coordinate systems used in this study. The Hill coordinate system was typically used to express dynamics around Ryugu. As shown in Fig. 2(a), the origin was the center of Ryugu. The $x$-axis was defined along the anti-solar line and the $z$-axis was taken as normal to Ryugu's orbital plane. The $y$-axis was defined to complete the right-handed coordinate system.

The Hayabusa2 spacecraft was predominantly operated

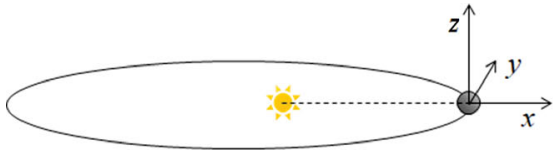

(a) Hill coordinate system at a hovering point where it was on a sub-Earth line at an altitude of $20 \mathrm{~km}$. Hence, the spacecraft's operation was easy to describe, given the special coordinates for Hayabusa2; i.e., the home position (HP) coordinate system. Figure 2(b) shows the HP coordinate system. The origin was the center of Ryugu and the $z$-axis was defined along the sub-Earth line. The $x$-axis was defined so that the first quadrant of the $X-Z$ plane included the sub-solar line and the $y$-axis was defined to complete the right-handed coordinate system.

The asteroid body fixed coordinate system was convenient for calculating the gravitational acceleration. The origin was the center of Ryugu and the $z$-axis was defined along the rotation axis. In this study, the rover insertion conditions were expressed in the HP coordinate system, the equations of motion were predominantly expressed in the Hill coordinate system, and the gravitational acceleration was calculated in the asteroid body fixed coordinate system; this coordinate system required proper transformation [3].

\section{Dynamics}

\subsection{Augmented elliptic Hill three-body problem (AEH3BP)}

The equations of motion are given in this section. The orbit was numerically propagated in an augmented elliptic Hill three-body problem (AEH3BP) according to the equations below. These equations were derived by extending the Hill three-body problem from the previous study [4].

$$
\begin{aligned}
x^{\prime \prime}-2 y^{\prime} & =\frac{a_{\mathrm{G} x}+\beta+3 x}{1+e_{\mathrm{r}} \cos f_{\mathrm{r}}} \\
y^{\prime \prime}+2 x^{\prime} & =\frac{a_{\mathrm{G} y}}{1+e_{\mathrm{r}} \cos f_{\mathrm{r}}} \\
z^{\prime \prime}+z & =\frac{a_{\mathrm{G} z}}{1+e_{\mathrm{r}} \cos f_{\mathrm{r}}}
\end{aligned}
$$

where the orbital angular velocity is normalized as unity, "' ' denotes the true anomaly derivative and $\boldsymbol{a}_{\mathrm{G}}=$ $\left[a_{\mathrm{G} x}, a_{\mathrm{G} y}, a_{\mathrm{G} z}\right]$ is normalized gravitational acceleration.

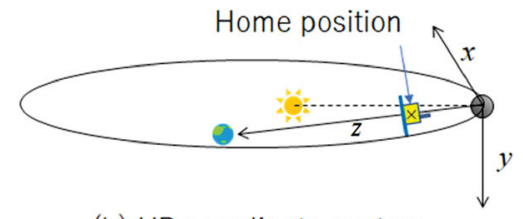

(b) HP coordinate system

Fig. 2 Hill coordinate system and HP coordinate system. 
These equations are normalized by both the distance (LU) and time unit. Both units were derived using the distance between the Sun and Ryugu as shown in Eq. (4), and are expressed by Eqs. (5) and (6), respectively.

$$
\begin{gathered}
d=\frac{a_{\mathrm{r}}\left(1-\mathrm{e}_{\mathrm{r}}^{2}\right)}{1+e_{\mathrm{r}} \cos f_{\mathrm{r}}} \\
\mathrm{LU}=d\left(\frac{\mu}{\mu_{\mathrm{S}}}\right)^{\frac{1}{3}} \\
\mathrm{TU}=\sqrt{\frac{d^{3}}{\mu_{\mathrm{S}}\left(1+e_{\mathrm{r}} \cos f_{\mathrm{r}}\right)}}
\end{gathered}
$$

where $d$ denotes distance between the Sun and asteroid, $a_{\mathrm{r}}, e_{\mathrm{r}}$, and $f_{\mathrm{r}}$ denote semi-major axis, eccentricity, and true anomaly of asteroid's orbit, respectively, and $\mu_{\mathrm{S}}$ and $\mu$ denote gravitational parameters of the Sun and asteroid. The equations of motion also include the normalized acceleration of solar radiation pressure (SRP) as $\beta$, expressed by Eq. (7). The $\beta$ value is zero when the rover is in Ryugu's shadow.

$\beta= \begin{cases}\frac{(1+\gamma) p_{0}}{(m / A) \mu_{\mathrm{S}}^{2 / 3} \mu^{1 / 3}} & \text { (the rover is exposed to the Sun) } \\ 0 & \text { (the rover is in the asteroid's shadow) }\end{cases}$

where $\gamma$ denotes reflectivity, $p_{0}=1.0197 \mathrm{~kg} \cdot \mathrm{m} / \mathrm{s}^{2}$ denotes solar flux, $m$ denotes mass of the rover, and $A$ denotes projected area of the rover. The normalized gravity potential, $U$ is the gravity potential associated with the asteroid harmonics and indicated by the following equation, using the Legendre polynomial $P_{l m}$ and Stockes coefficients $C_{l m}$ and $S_{l m}$.

$$
\begin{aligned}
U(r, \phi, \lambda)= & \frac{1}{r} \sum_{l=0}^{\infty} \sum_{m=0}^{l}\left(\frac{R}{r}\right)^{l} P_{l m}(\sin \phi)\left(C_{l m} \cos m \lambda\right. \\
& \left.+S_{l m} \sin m \lambda\right)
\end{aligned}
$$

where $R$ is the reference radius and $\phi$ and $\lambda$ are the latitude and longitude, respectively. $\phi$ and $\lambda$ are defined in the asteroid body fixed coordinate system. The reference radius $R$ is the normalized value of $526 \mathrm{~m}$, from Eq. (5). The gravitational acceleration is calculated from the gravity potential as follows:

$$
\overline{\boldsymbol{a}_{\mathrm{G}}}=\frac{\partial U}{\partial \overline{\boldsymbol{r}}}
$$

where $\overline{\boldsymbol{a}_{\mathrm{G}}}$ and $\overline{\boldsymbol{r}}$ are the gravitational acceleration and position vector expressed in the asteroid body fixed coordinate system, respectively. The gravitational acceleration in the Hill coordinate system, $\boldsymbol{a}_{\mathrm{G}}$, can be obtained by transforming the coordinate system of $\overline{\boldsymbol{a}_{\mathrm{G}}}$. Tables 1 and 2 list the physical parameters of Rover 2 and Ryugu. These parameters are used in Eqs. (1)-(8).

Table 1 MINERVA II-2 parameters

\begin{tabular}{ccc}
\hline Item & Symbol & Value \\
\hline Mass & $m$ & $0.877 \mathrm{~kg}$ \\
Projected area & $A$ & $0.02175 \mathrm{~m}^{2}$ \\
Reflectivity & $\gamma$ & 0.35 \\
\hline
\end{tabular}

Table 2 Ryugu parameters

\begin{tabular}{cc}
\hline Item & Value \\
\hline Gravitational constant & $30 \mathrm{~m}^{3} / \mathrm{s}^{2}$ \\
Eccentricity & 0.190 \\
Equatorial radius & $503 \mathrm{~m}$ \\
Polar radius & $443 \mathrm{~m}$ \\
Pole direction & $179.3,-87.44] \mathrm{deg}$ \\
Obliquity & $171.64 \mathrm{deg}$ \\
Rotational period & $7.6326 \mathrm{~h}$ \\
\hline
\end{tabular}

\subsection{Asteroid's gravity and shape model}

This section describes the gravity and shape models of Ryugu used in this study. The Hayabusa2 mission team constructed a polygonal asteroid shape model before touchdown, as shown in Fig. 3(b). The previous study developed the gravitational acceleration, computed using the homogeneous density polyhedron model [5]. In addition, the Stokes coefficients $C_{l m}$ and $S_{l m}$ have already been directly converged using the polyhedron gravity model [6]. These two gravity models have advantages and disadvantages. Although the calculation accuracy using the polyhedron gravity model is high, the calculation cost is also high. Conversely, the calculation cost of using the spherical harmonics model is lower than that of the polyhedron model. However, the accuracy usually reduces when the probe is close to the surface. Therefore, this study used both gravity models. After the spherical harmonics gravity model was used for searching the solution space, the polyhedron gravity model was used for validating the orbit candidate selected by the grid search.

Figure 3 shows the two shape models of Ryugu in the Hill coordinate system at 16:00:00 on 2 October 2019, when we planned to release the rover. The magenta lines indicate the rotation axes. As shown in Fig. 3, the rotation axis was almost perpendicular to the $X-Y$ 


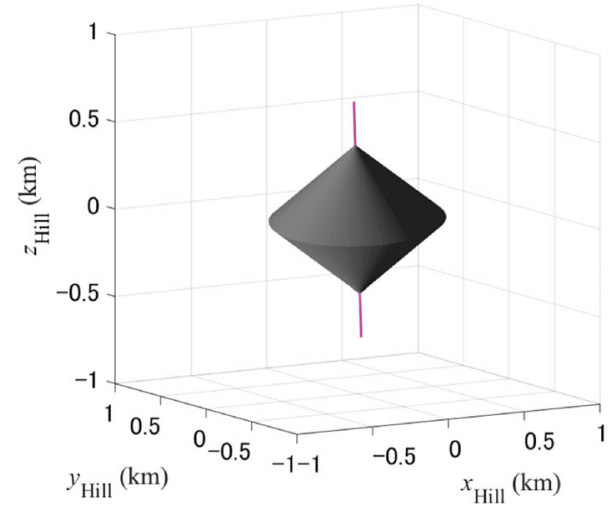

(a) Two-cone shape model

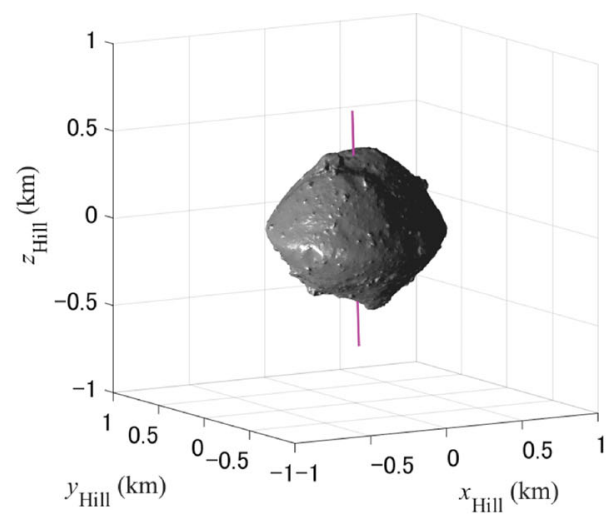

(b) 46874 polygon shape model

Fig. 3 Asteroid models.

plane. Note that the obliquity was $171.64 \mathrm{deg}$, according to Table 2, which is almost a completely retrograde rotation; when the obliquity is $180 \mathrm{deg}$, the rotation direction is retrograde. When the spherical harmonics gravity model was used, the shape was set to be two cones, as shown in Fig. 3(a). The shape models were used to determine whether the rover collides with the surface. Note that $8 \times 8$ spherical harmonics gravity is taken into account and the Stokes coefficients are listed in Table 3. Table 3 clarifies the strong oblateness of Ryugu as $C_{20}$ is of order $10^{-2}$, which is much larger than the other terms.

\subsection{Rover 2 deployment}

Rover 2 was ejected in the $-y_{\mathrm{SC}}$ direction, in spacecraft body fixed coordinates. This nominal release direction was the same as the $-y_{\mathrm{HP}}$ direction in the HP coordinates because the $x_{\mathrm{SC}}$ and $y_{\mathrm{SC}}$ axes corresponded to $x_{\mathrm{HP}}$ and $y_{\mathrm{HP}}$ when the spacecraft maintained constant attitude; this occurred during the release of MINERVA II-2 to guarantee both communication with Earth and power generation. As shown in Fig. 4, the nominal release direction was inclined $5 \mathrm{deg}$ in the direction of $-z_{\mathrm{SC}}$ to avoid impact with the spacecraft. Most significantly, this release velocity had a large uncertainty of $\pm 10 \mathrm{~cm} / \mathrm{s}$. The next section describes how this initial deployment error was overcome and the rover was inserted into a stable orbit around Ryugu.

\section{Orbit design}

\subsection{Critical velocity}

We first investigated the zero velocity curve around Ryugu to approximately determine the orbit insertion
Table 3 Unnormalized gravity coefficients

\begin{tabular}{|c|c|c|c|}
\hline$l$ & $m$ & $C_{l m}$ & $S_{l m}$ \\
\hline 0 & 0 & $1.000000000 \mathrm{e}+00$ & $0.000000000 \mathrm{e}+00$ \\
\hline 1 & 0 & $-2.599033996 \mathrm{e}-16$ & $0.000000000 \mathrm{e}+00$ \\
\hline 1 & 1 & $1.062577084 \mathrm{e}-16$ & $3.494929332 \mathrm{e}-16$ \\
\hline 2 & 0 & $-3.898812629 \mathrm{e}-02$ & $0.000000000 \mathrm{e}+00$ \\
\hline 2 & 1 & $-9.356634314 \mathrm{e}-05$ & $-5.918281151 \mathrm{e}-04$ \\
\hline 2 & 2 & $-1.579451669 \mathrm{e}-03$ & $-1.099248649 \mathrm{e}-03$ \\
\hline 3 & 0 & $1.808135634 \mathrm{e}-03$ & $0.000000000 \mathrm{e}+00$ \\
\hline 3 & 1 & $2.903207922 \mathrm{e}-03$ & $3.092943141 \mathrm{e}-03$ \\
\hline 3 & 2 & $5.316500199 \mathrm{e}-04$ & $-1.296893281 \mathrm{e}-03$ \\
\hline 3 & 3 & $1.380156630 \mathrm{e}-04$ & $-2.853838179 \mathrm{e}-04$ \\
\hline 4 & 0 & $2.235039289 \mathrm{e}-02$ & $0.000000000 \mathrm{e}+00$ \\
\hline 4 & 1 & $2.174354306 \mathrm{e}-03$ & $9.805267670 \mathrm{e}-04$ \\
\hline 4 & 2 & $-7.205712007 \mathrm{e}-05$ & $3.969527686 \mathrm{e}-05$ \\
\hline 4 & 3 & $-7.624624543 \mathrm{e}-06$ & $-9.146659147 \mathrm{e}-06$ \\
\hline 4 & 4 & $-1.216141238 \mathrm{e}-05$ & $-1.639308253 \mathrm{e}-06$ \\
\hline 5 & 0 & $1.679282696 \mathrm{e}-04$ & $0.000000000 \mathrm{e}+00$ \\
\hline 5 & 1 & $3.656499615 \mathrm{e}-04$ & $2.584972684 \mathrm{e}-04$ \\
\hline 5 & 2 & $6.123689211 \mathrm{e}-06$ & $9.739625836 \mathrm{e}-05$ \\
\hline 5 & 3 & $-1.515033208 \mathrm{e}-05$ & $2.531872788 \mathrm{e}-06$ \\
\hline 5 & 4 & $-1.539966499 \mathrm{e}-06$ & $-2.295906186 \mathrm{e}-06$ \\
\hline 5 & 5 & $1.914132501 \mathrm{e}-08$ & $-2.029363817 \mathrm{e}-07$ \\
\hline 6 & 0 & $-7.777325445 \mathrm{e}-03$ & $0.000000000 \mathrm{e}+00$ \\
\hline 6 & 1 & $-7.138750680 \mathrm{e}-04$ & $3.514570437 \mathrm{e}-04$ \\
\hline 6 & 2 & $-4.676504321 \mathrm{e}-05$ & $-1.542343672 \mathrm{e}-05$ \\
\hline 6 & 3 & $-2.590314773 \mathrm{e}-06$ & $6.287585904 \mathrm{e}-06$ \\
\hline 6 & 4 & $-6.060159865 \mathrm{e}-08$ & $6.403054172 \mathrm{e}-08$ \\
\hline 6 & 5 & $3.780898045 \mathrm{e}-08$ & $4.947584575 \mathrm{e}-08$ \\
\hline 6 & 6 & $7.137350504 \mathrm{e}-08$ & $3.878680629 \mathrm{e}-08$ \\
\hline 7 & 0 & $-4.164003942 \mathrm{e}-04$ & $0.000000000 \mathrm{e}+00$ \\
\hline 7 & 1 & $1.446753633 \mathrm{e}-04$ & $3.337757330 \mathrm{e}-04$ \\
\hline 7 & 2 & $1.963966097 \mathrm{e}-05$ & $-3.371212653 \mathrm{e}-05$ \\
\hline 7 & 3 & $4.651910495 \mathrm{e}-07$ & $-2.334143802 \mathrm{e}-06$ \\
\hline 7 & 4 & $-1.617124235 \mathrm{e}-07$ & $-1.377487760 \mathrm{e}-07$ \\
\hline 7 & 5 & $-6.548581422 \mathrm{e}-08$ & $2.379569051 \mathrm{e}-08$ \\
\hline 7 & 6 & $1.755518584 \mathrm{e}-09$ & $4.088822413 \mathrm{e}-10$ \\
\hline 7 & 7 & $-6.728838670 \mathrm{e}-10$ & $2.736666545 \mathrm{e}-09$ \\
\hline 8 & 0 & $3.729550481 \mathrm{e}-03$ & $0.000000000 \mathrm{e}+00$ \\
\hline 8 & 1 & $1.605378972 \mathrm{e}-04$ & $2.789848182 \mathrm{e}-05$ \\
\hline 8 & 2 & $-5.411124027 \mathrm{e}-06$ & $2.791469736 \mathrm{e}-06$ \\
\hline 8 & 3 & $2.341238678 \mathrm{e}-07$ & $-1.061777400 \mathrm{e}-06$ \\
\hline 8 & 4 & $-6.925824555 \mathrm{e}-08$ & $2.885921386 \mathrm{e}-08$ \\
\hline 8 & 5 & $1.354088745 \mathrm{e}-08$ & $-1.175582598 \mathrm{e}-08$ \\
\hline 8 & 6 & $-3.672817326 \mathrm{e}-09$ & $-3.176220530 \mathrm{e}-09$ \\
\hline 8 & 7 & $3.819611085 \mathrm{e}-10$ & $-5.905042470 \mathrm{e}-10$ \\
\hline 8 & 8 & $1.894731930 \mathrm{e}-11$ & $-2.034058704 \mathrm{e}-10$ \\
\hline
\end{tabular}




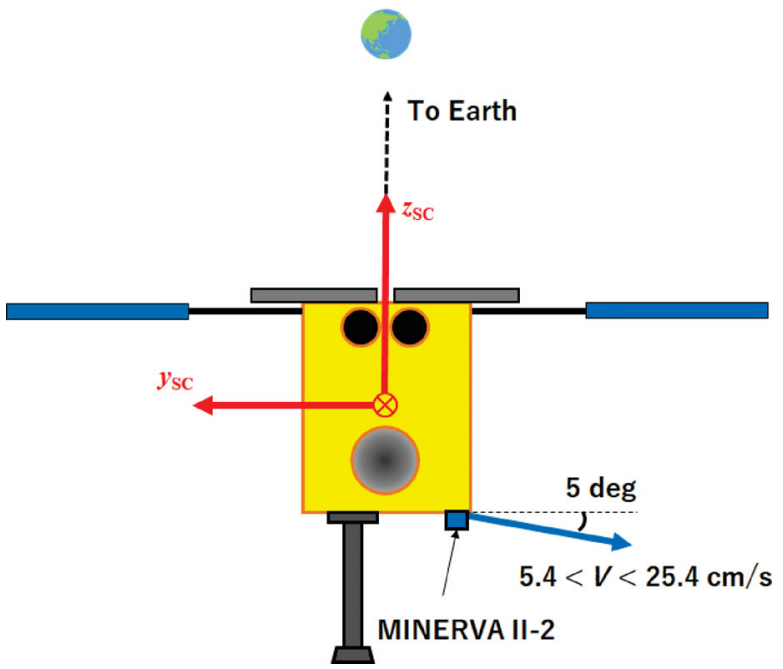

Fig. 4 MINERVA II-2 deployment.

policy. Since the zero velocity curve cannot be defined as a time-invariant parameter in the EH3BP, we introduced the quasi-elliptic Hill three-body problem (QEH3BP), which is the modified equations of EH3BP developed by the previous study [7]. The Jacobi integral can be defined in QEH3BP as the QEH3BP equations are the autonomous equations of motion. The normalized QEH3BP equations are expressed as follows:

$$
\begin{gathered}
\ddot{x}-2 \tilde{n} \dot{y}=-\frac{x}{r^{3}}+\beta-\frac{3}{1+e_{\mathrm{r}} \cos f_{\mathrm{r}}} \tilde{n} x \\
\ddot{y}+2 \tilde{n} \dot{x}=-\frac{y}{r^{3}} \\
\ddot{z}=-\frac{z}{r^{3}}-\tilde{n}^{2} z
\end{gathered}
$$

where the gravity is approximated by a point mass. $\tilde{n}$ is indicated by Eq. (13).

$$
\tilde{n}=\frac{\left(1+e_{\mathrm{r}} \cos f_{\mathrm{r}}\right)^{2}}{\left(1-e^{2}\right)^{\frac{3}{2}}}
$$

The conserved quantity (i.e., Jacobi integral) can be calculated in QEH3BP as follows:

$$
\begin{aligned}
& C_{\mathrm{J}}=\frac{v^{2}}{2}+\bar{U} \\
& \bar{U}=-\frac{1}{2} \tilde{n}^{2}\left(\frac{3 x^{2}}{1+e_{\mathrm{r}} \cos f_{\mathrm{r}}}-z^{2}\right)-\frac{1}{r}-\beta x
\end{aligned}
$$

Figure 5 shows the unnormalized zero velocity curve obtained by substituting $v=0$ into Eq. (14). The epoch was set to be 2 October 2019, at 16:00:00. There is one Lagrange point (L2) because the L1 point disappears due to SRP force. Since the bold line in Fig. 5 indicates the closed region of $C_{\mathrm{J}}$, proximity to Ryugu was not

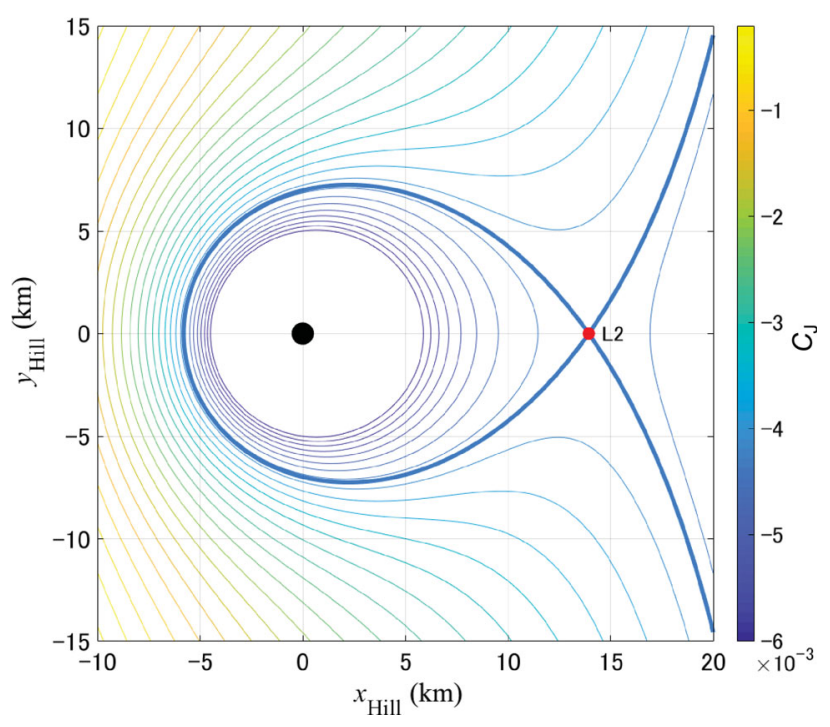

Fig. 5 Zero velocity curve.

guaranteed outside of the bold line. Thus, the rover required release inside this closed region. The critical velocity $\left(v_{\mathrm{c}}\right)$ that guaranteed the rover's proximity to Ryugu can be defined as follows:

$$
v_{\mathrm{c}}=\sqrt{2\left(C_{\mathrm{J}}^{*}-\bar{U}\right)}
$$

$C_{\mathrm{J}}^{*}$ corresponds to the bold line in Fig. 5. The sum of the rover's orbital velocity, $v_{\mathrm{o}}$, release velocity error must be smaller than $v_{\mathrm{c}}$. Therefore, the velocity margin $\left(v_{\mathrm{m}}\right)$ that guarantees not escaping from Ryugu could be obtained in the cases where the rover was inserted in the tangential and normal direction to the orbit.

$$
v_{\mathrm{m}}= \begin{cases}v_{\mathrm{c}}-v_{\mathrm{o}} & (\text { tangential }) \\ \sqrt{v_{\mathrm{c}}^{2}-v_{\mathrm{o}}^{2}} & \text { (normal) }\end{cases}
$$

When the spacecraft was on the sub-Earth line and $v_{\mathrm{o}}$ was calculated as the circular orbital velocity in the twobody problem and expressed by $v_{\mathrm{o}}=\sqrt{\mu / r}$, the relation of $v_{\mathrm{m}}$ and orbital radius is shown in Fig. 6. Obviously, the rover requires release in the normal direction to the orbit as the velocity margin of the normal direction is more than twice as large as that of the tangential direction. In addition, Fig. 6 indicates that an orbit radius smaller than $1.5 \mathrm{~km}$ should be chosen because the rover release velocity has $\pm 0.1 \mathrm{~m} / \mathrm{s}$ error.

\subsection{Terminator orbit and equatorial orbit}

In this study, there were two candidate orbits into which the rover could be inserted: a terminator orbit and equatorial orbit. This subsection compares the 


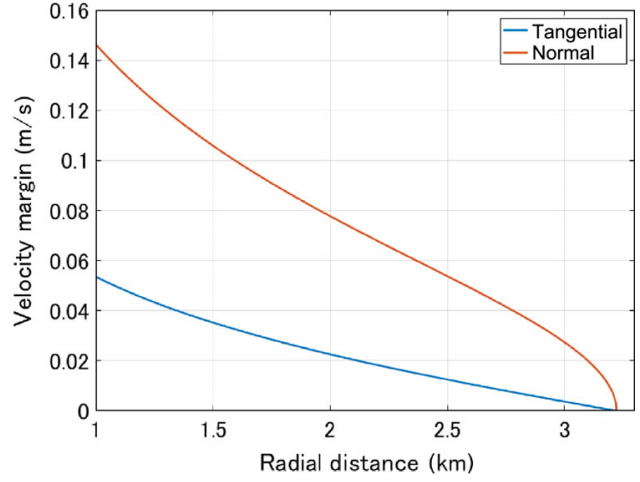

Fig. 6 Velocity margin.

initially circular terminator orbit and the initially circular equatorial orbit. The terminator orbit is a well-known stable frozen orbit around a small body in an environment strongly perturbed by the SRP force. Many previous papers have investigated the characteristics and stability of the terminator orbit; in addition to being stable inside the surrounding linearized region, there is a broad solution space that avoids both escape and impact, even if the orbits are outside the linearized region $[8,9]$.

Two initially circular terminator orbits with radial distances of 1.0 and $1.5 \mathrm{~km}$, respectively, were propagated over 20 days according to Eqs. (1)-(3), as shown in Fig. 7. These orbits are displayed in the Hill coordinate system. The nominal orbits, shown as blue lines, indicate initially circular terminator orbits. Other orbits include initial velocity errors of $\pm 10 \mathrm{~cm} / \mathrm{s}$ due to the release
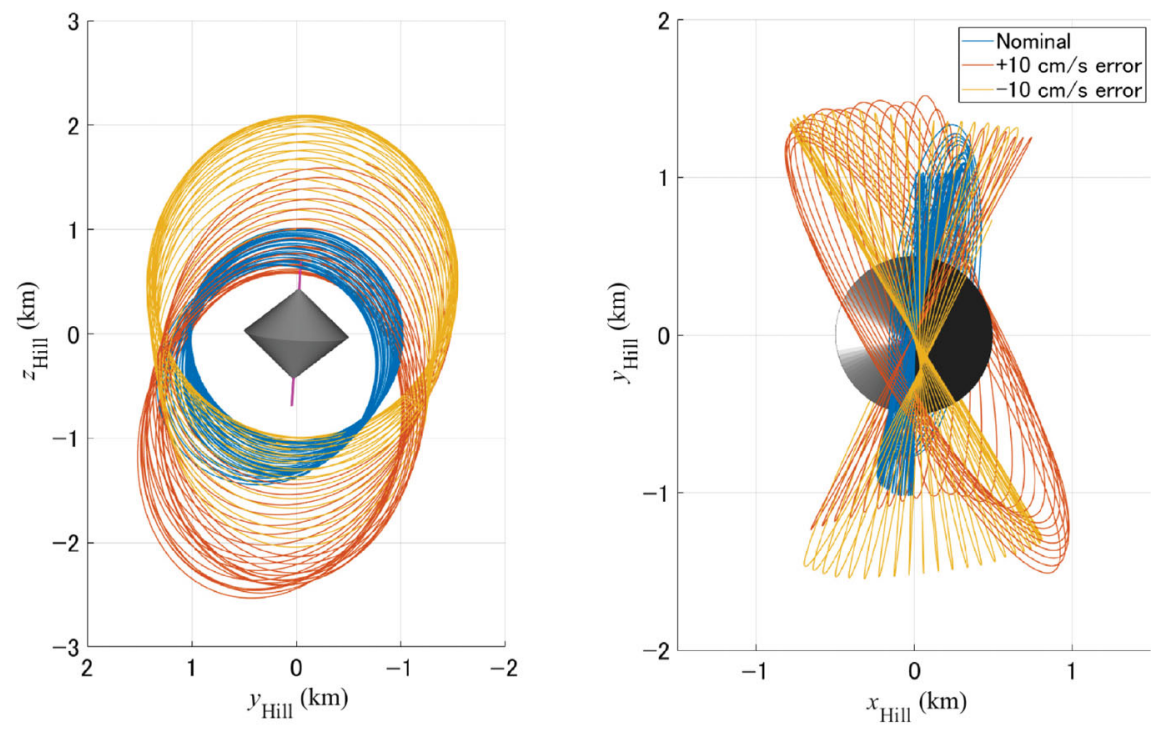

(a) $r=1.0 \mathrm{~km}$
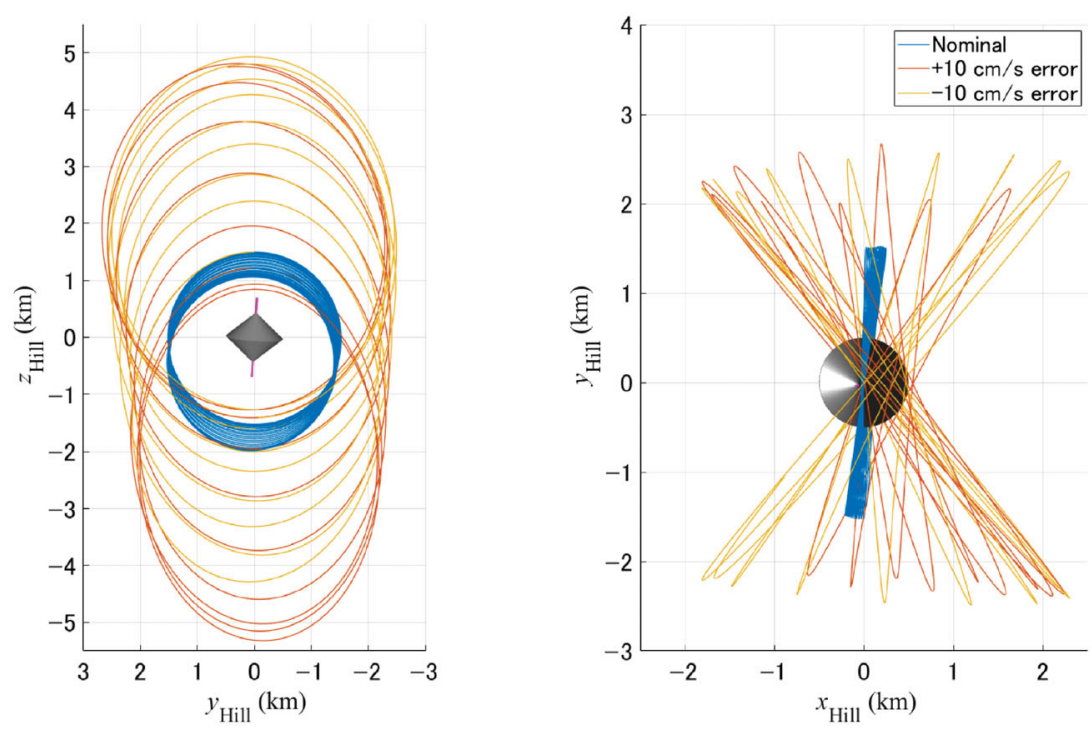

(b) $r=1.5 \mathrm{~km}$

Fig. 7 Terminator orbits. 
velocity of $15.4 \pm 10 \mathrm{~cm} / \mathrm{s}$. The initial position was set to be $\left[x_{\mathrm{Hill}}, y_{\mathrm{Hill}}, z_{\mathrm{Hill}}\right]=[0,0,1.0$ or $1.5 \mathrm{~km}]$ and the velocity errors were given to $v_{x}$. Figure 7 shows that the terminator orbits with large velocity uncertainties were stable over more than 20 days, even if perturbations due to higher order gravity and SRP were included in the dynamics around Ryugu. Figure 8 shows the history of the eccentricity of the terminator orbits. Despite the eccentricities oscillating in the case of $\pm 10 \mathrm{~cm} / \mathrm{s}$, the rover avoids impacting with the surface.

An alternative candidate is the near-equatorial orbit around Ryugu. It is known that there are stable equatorial orbits when the $J_{2}$ terms of gravity and SRP are dominant in the vicinity of Ryugu. These are frozen orbits, sometimes called heliotropic orbits by previous studies $[10,11]$. Since Ryugu also has large oblateness, the eccentricity of the equatorial orbits around Ryugu tend towards stability. Figure 9 shows the initially

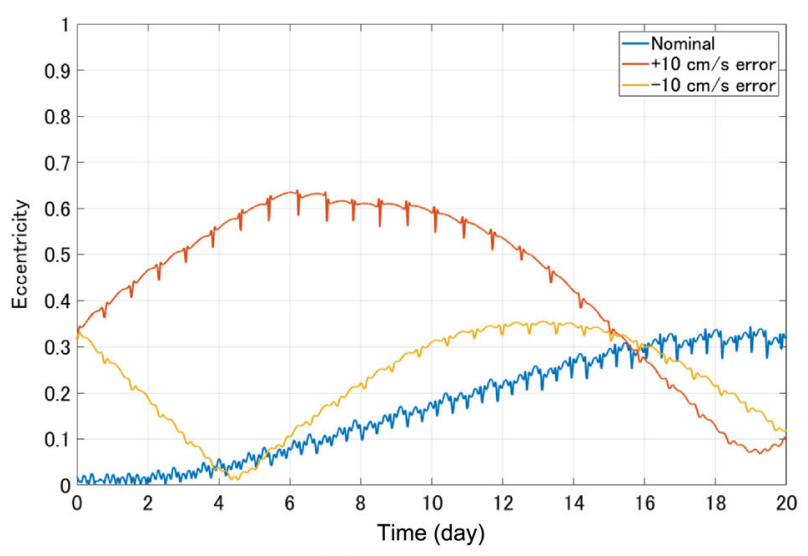

(a) $r=1.0 \mathrm{~km}$

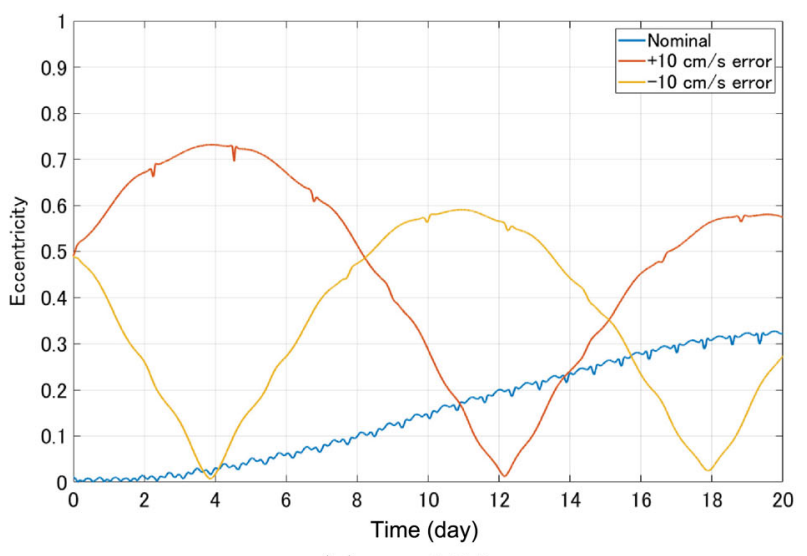

(b) $r=1.5 \mathrm{~km}$

Fig. 8 Eccentricity of terminator orbits.
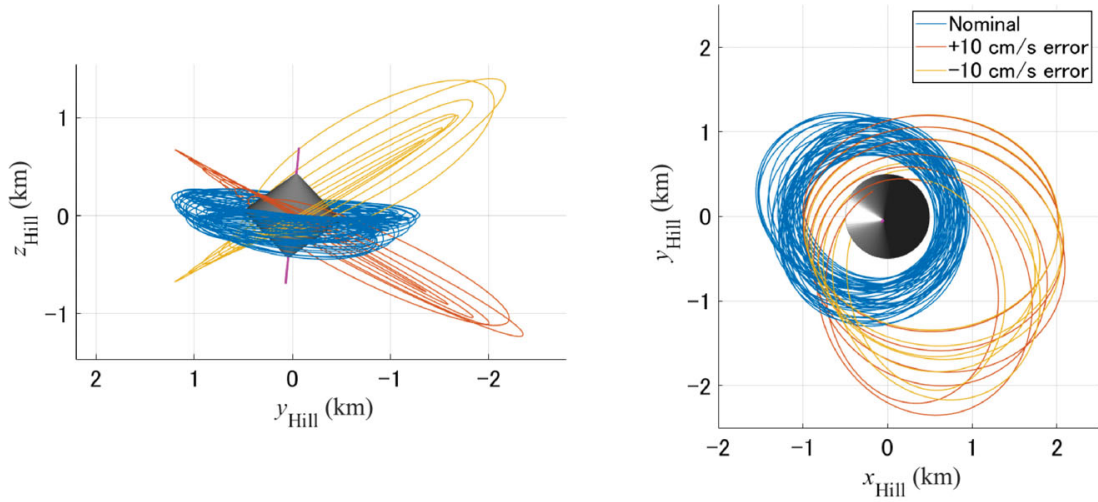

(a) $r=1.0 \mathrm{~km}$
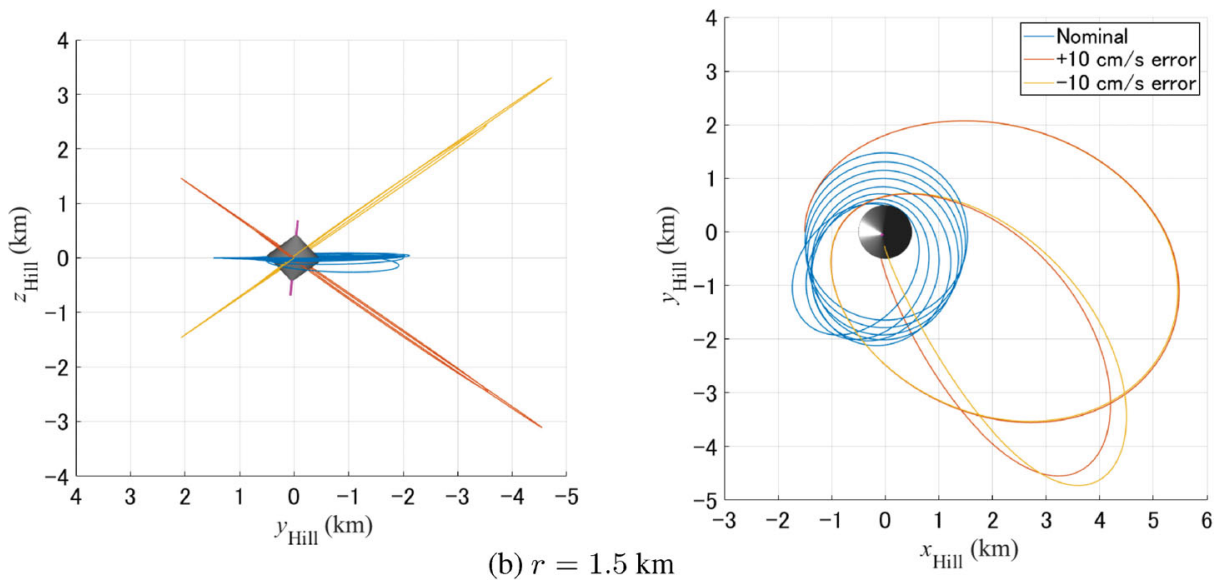

(b) $r=1.5 \mathrm{~km}$

Fig. 9 Equatorial orbits. 
circular orbits as the nominal orbits in the two cases where the radial distance is equivalent to 1.0 and $1.5 \mathrm{~km}$ in Hill coordinates. These were propagated for 20 days. The initial position was set to be $\left[x_{\mathrm{Hill}}, y_{\mathrm{Hill}}, z_{\mathrm{Hill}}\right]=[-1.0$ or $-1.5,0,0 \mathrm{~km}]$ and the velocity errors were given to $v_{z}$ as $10 \mathrm{~cm} / \mathrm{s}$. Although the rover finally impacts with the surface, except for the nominal case of $r=1.0 \mathrm{~km}$, it does not escape from Ryugu; even if the initial velocities include an error of $10 \mathrm{~cm} / \mathrm{s}$. The eccentricities are shown in Fig. 10 and the minimum duration is 5 days. These results indicate that the equatorial orbit is also a valid candidate for the orbit owing to its relative stability, considering the gravitational irregularity.

In this section, we compare the orbits from the standpoint of Rover 2 insertion operability. Figure 11 shows a comparison of insertion into the terminator orbit and equatorial orbit. In both orbits, the spacecraft must decrease its altitude to insert Rover 2. If the equatorial orbit is chosen, after the spacecraft descends along the sub-Earth line the spacecraft should give the rover's orbit velocity and correction maneuver in the

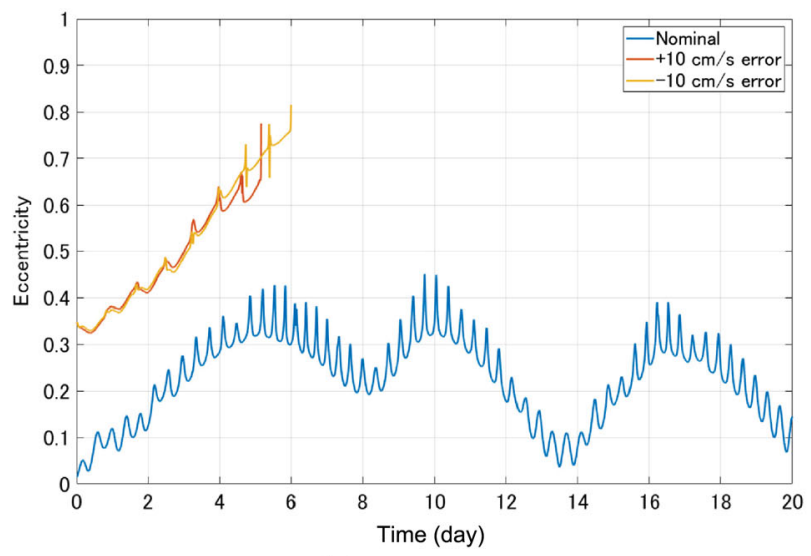

(a) $r=1.0 \mathrm{~km}$
$-z_{\mathrm{Hill}}$ direction because the release direction is $+z_{\mathrm{Hill}}$. Since this operation can ensure release direction normal to the orbital velocity, the orbit becomes robust against release velocity error. Conversely, if the terminator orbit is chosen, the spacecraft must move to the polar position and insert the rover so that the release direction corresponds to the direction normal to the orbital velocity, without any change in attitude. This extremely unusual operation is more difficult than that of the equatorial orbit as the spacecraft must be very far from the sub-earth line. Therefore, from the standpoint of operational safety the equatorial orbit is better than the terminator orbit for the Hayabusa2 spacecraft.

\subsection{Solution space}

To determine the best initial conditions for inserting Rover 2 into equatorial orbit, we conducted a grid search of the orbits' solution space around Ryugu. Initial velocities were given in HP coordinates and $v_{x \mathrm{HP}}$ and $v_{y \mathrm{HP}}$ were chosen as grid search parameters. $v_{z \mathrm{HP}}$ was set to 0 . Initial positions were on the sub-Earth

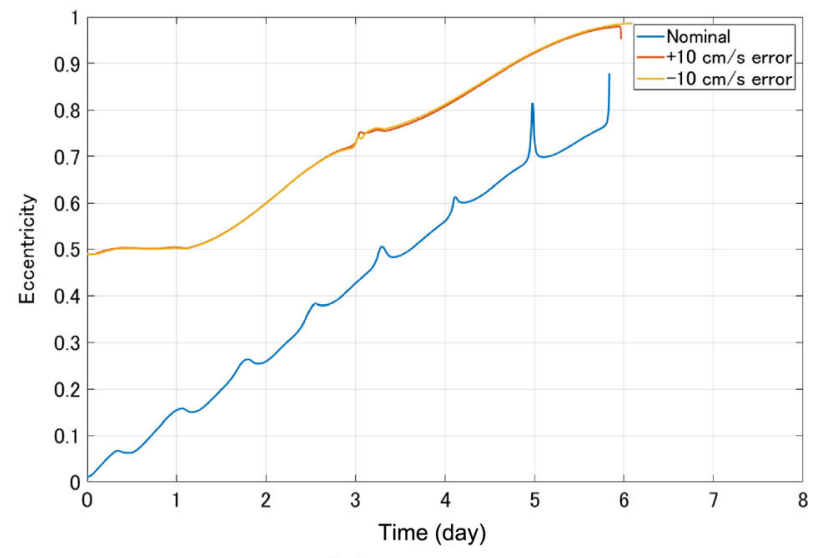

(b) $r=1.5 \mathrm{~km}$

Fig. 10 Eccentricity of equatorial orbits.

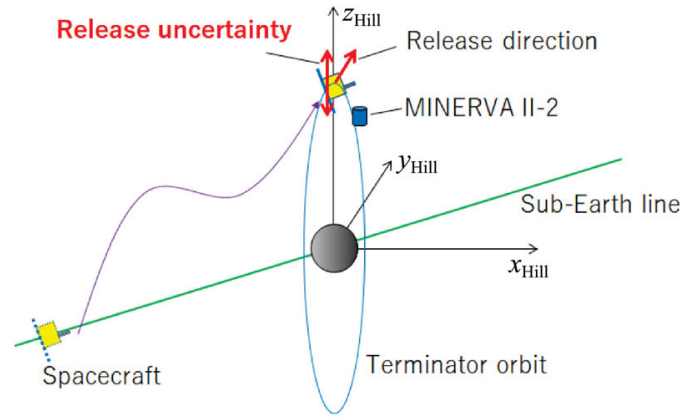

(a) Insertion into terminator orbit

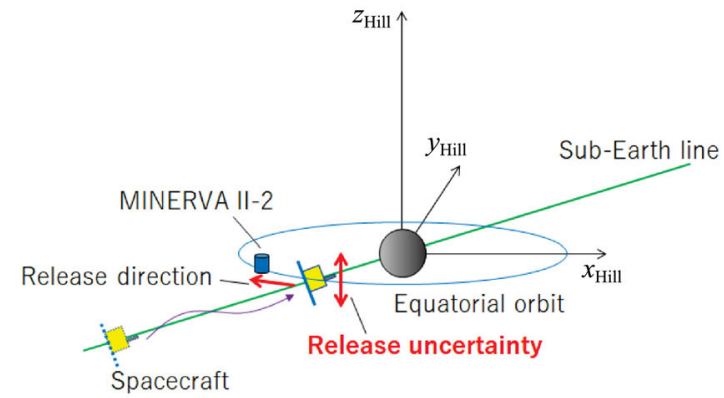

(b) Insertion into equatorial orbit

Fig. 11 Comparison of MINERVA II-2's insertion operation. 
line and the radial distances were varied from 1.0 to $3.0 \mathrm{~km}$. Figures 12 and 13 show the solution space of duration and number of periods, respectively. The white areas indicate that the rover escapes from Ryugu and the areas surrounded by red lines indicate that the rover impacts with the surface without any revolutions. Note that the areas where $v_{y \mathrm{HP}}$ is close to zero correspond to the equatorial orbits because the $y_{\text {HP }}$ direction corresponds to the $z_{\text {Hill }}$ direction. These figures show that the larger the orbit becomes, the smaller the solution space becomes. Moreover, $v_{y \mathrm{HP}}$ should be chosen as zeros because the solution spaces are symmetric about $v_{y \mathrm{HP}}$. It is important that $v_{y \mathrm{HP}}$ has a $10 \mathrm{~cm} / \mathrm{s}$ velocity margin in the valid solution area that avoids both escape and impact without revolution.

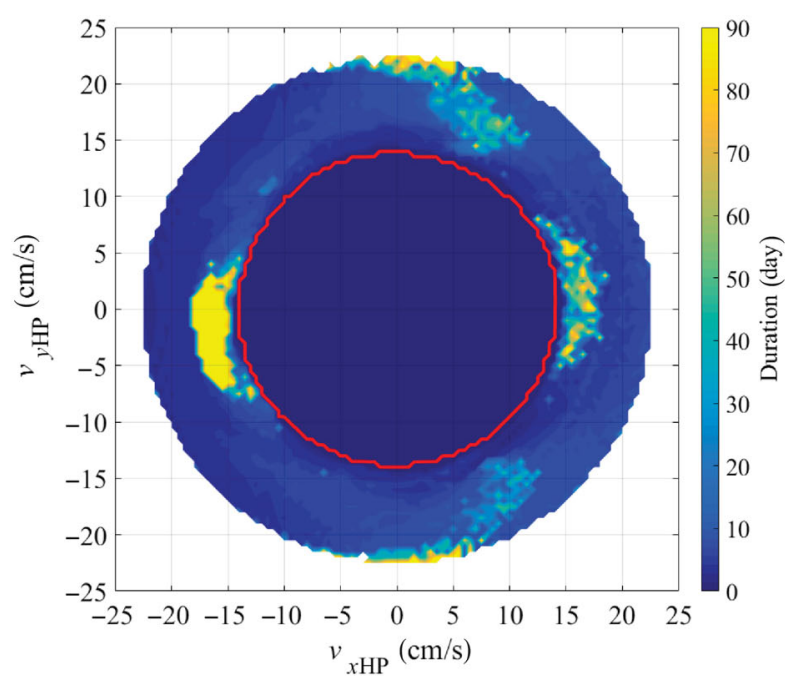

(a) $r=1.0 \mathrm{~km}$

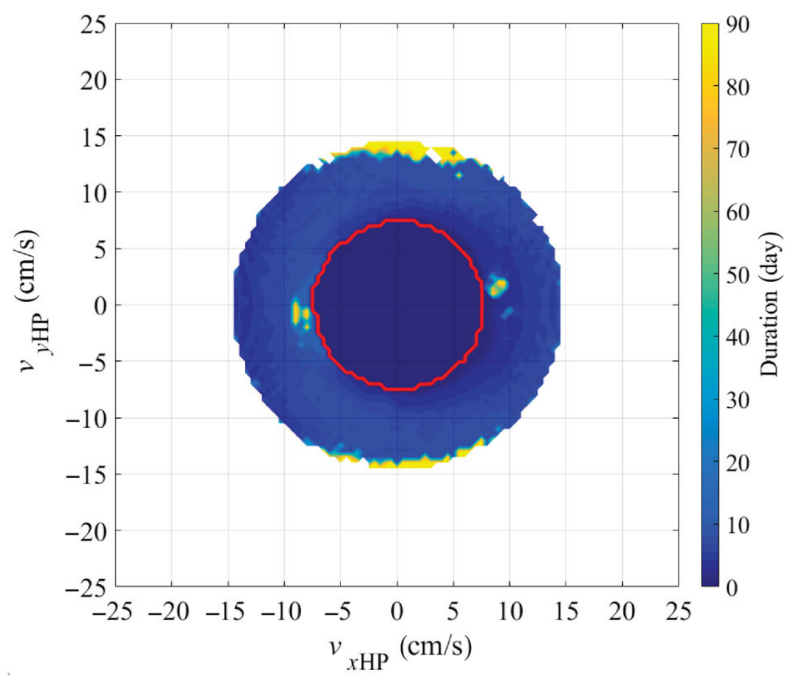

(c) $r=2.0 \mathrm{~km}$
These figures reveal that with the exception of (d) $r=3.0 \mathrm{~km}$, the solution case satisfies the $10 \mathrm{~cm} / \mathrm{s}$ $v_{y \mathrm{HP}}$ margin and as the the orbit becomes smaller the $v_{y \mathrm{HP}}$ margin becomes accordingly larger. Although $r=1.0 \mathrm{~km}$ is optimal in terms of orbit robustness against velocity error, it is a high risk operation; $r=1.0$ $\mathrm{km}$ indicates the spacecraft altitude is less than $500 \mathrm{~m}$. Hence, we chose a value of $r=1.5 \mathrm{~km}$, accounting for both velocity margin and operational safety.

Another topic of interest concerns which orbit direction is optimal. There are two areas where the $v_{y \mathrm{HP}}$ margins are more than $10 \mathrm{~cm} / \mathrm{s}$ and $v_{x \mathrm{HP}}$ is from -14 to $-10 \mathrm{~cm} / \mathrm{s}$ and from 10 to $14 \mathrm{~cm} / \mathrm{s}$ on the left and right sides of Fig. 12, respectively. Each area corresponds to the prograde and retrograde orbits.

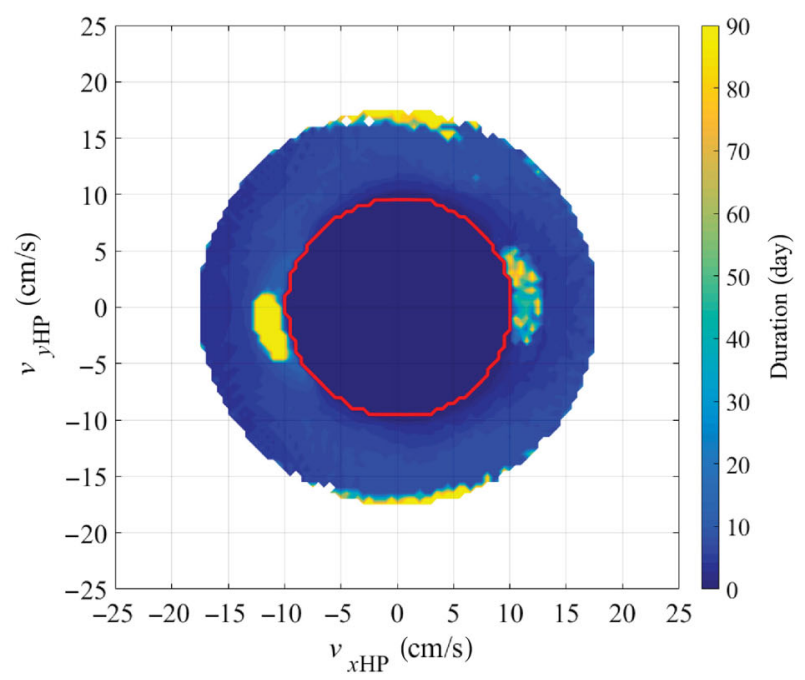

(b) $r=1.5 \mathrm{~km}$

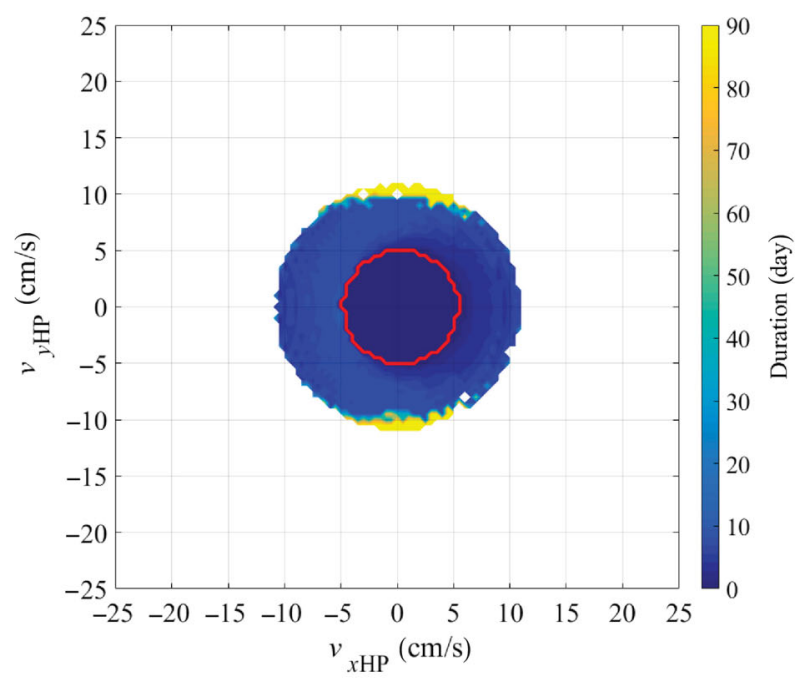

(d) $r=3.0 \mathrm{~km}$

Fig. 12 Duration map with several radial distances. 


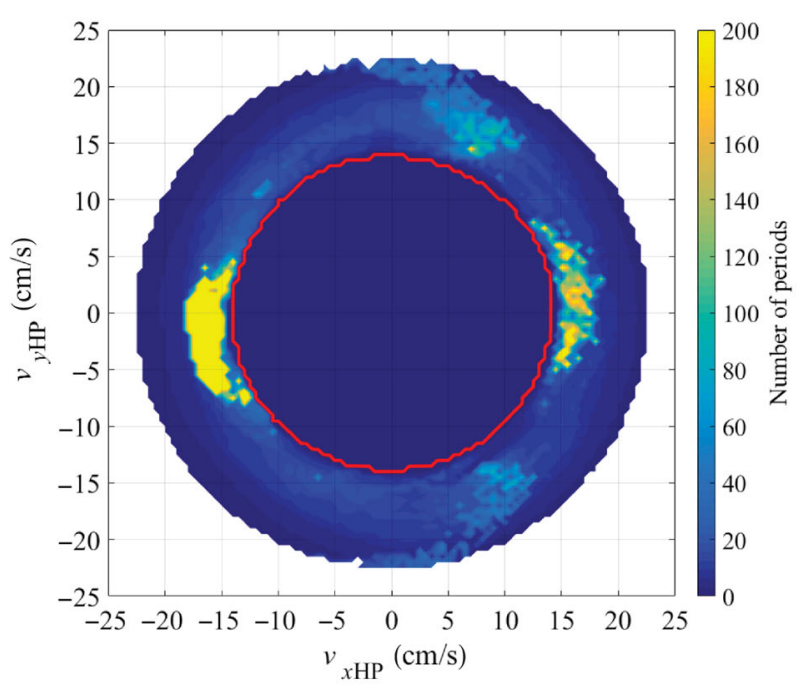

(a) $r=1.0 \mathrm{~km}$

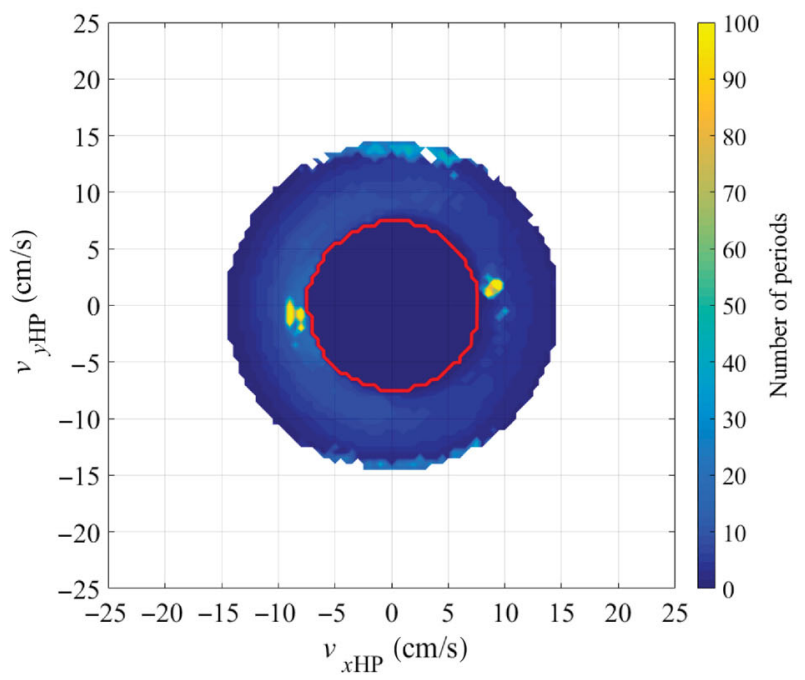

(c) $r=2.0 \mathrm{~km}$

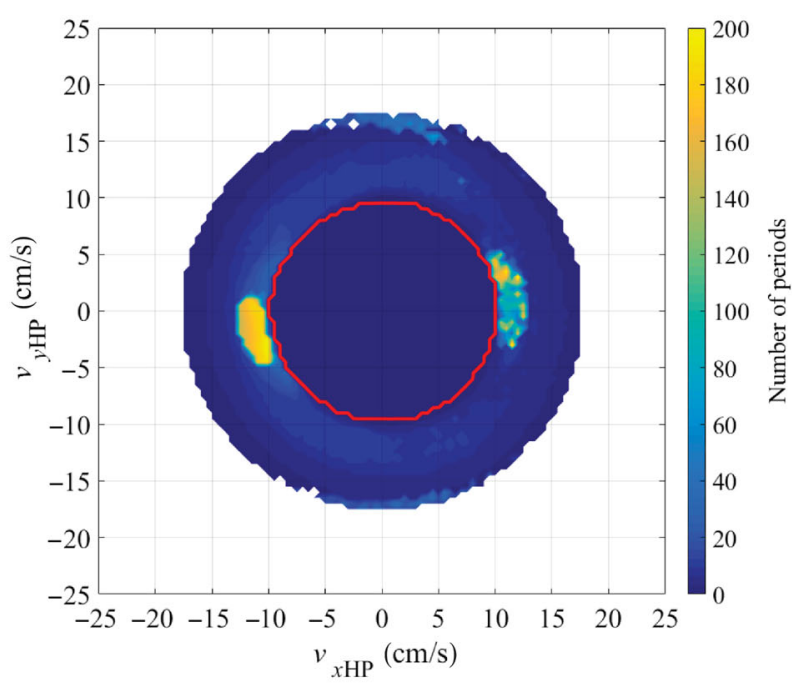

(b) $r=1.5 \mathrm{~km}$

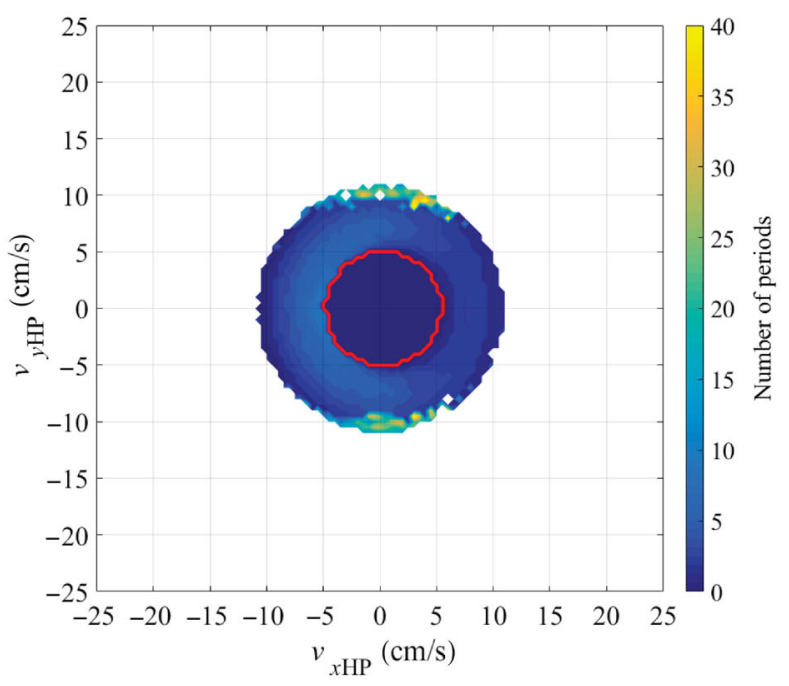

(d) $r=3.0 \mathrm{~km}$

Fig. 13 Number of periods map with several radial distances.

Note that "prograde" and "retrograde" are defined in Hill coordinates. Figure 14 shows enlarged maps of Fig. 12(b). Interestingly, these figures are very different. Prograde orbits have a broad yellow area, indicating a stable and long duration, whereas retrograde orbits have a mottled pattern that indicates an orbit family sensitive to errors. For instance, Fig. 15 shows the case of $\left[v_{x \mathrm{HP}}, v_{y \mathrm{HP}}\right]=[-0.113,0] \mathrm{m} / \mathrm{s}$ and $\left[v_{x \mathrm{HP}}, v_{y \mathrm{HP}}\right]=$ $[0.113,0] \mathrm{m} / \mathrm{s}$. This prograde orbit is very stable and has a duration of more than 90 days, whereas the retrograde orbit has a duration of less than 12 days. It is assumed that the reason for this derives from gravitational irregularity having a stronger effect on retrograde orbits than on prograde orbits as Ryugu's rotation is retrograde and in the same direction as the retrograde orbit. When an orbit direction opposing Ryugu's rotation is chosen, the perturbation averages out and the orbit tends towards stability. If the rover is not inserted into orbit but the spacecraft is, the orbit direction should oppose Ryugu's direction of rotation to ensure maximum stability. However, in this study the rover was required to finally land on the surface without an excessively long orbit duration, as mentioned in Section 1. Therefore, the somewhat unstable retrograde orbit was a more suitable choice for Rover 2's orbit.

\subsection{Monte Carlo simulation}

The previous subsection revealed that $v_{x \mathrm{HP}}$ should be a positive value for the retrograde orbit. We subsequently conducted a Monte Carlo simulation to 


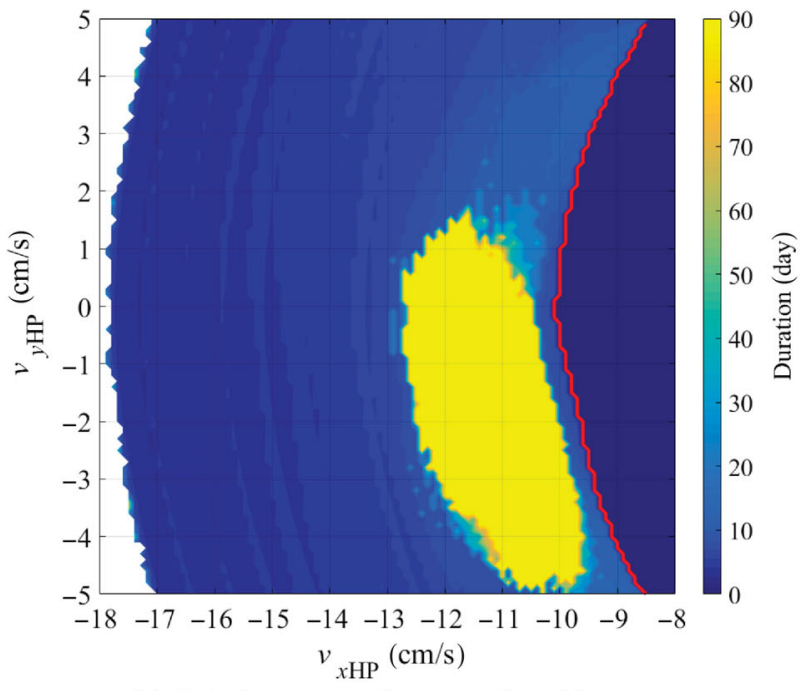

(a) Solution space of retrograde orbits

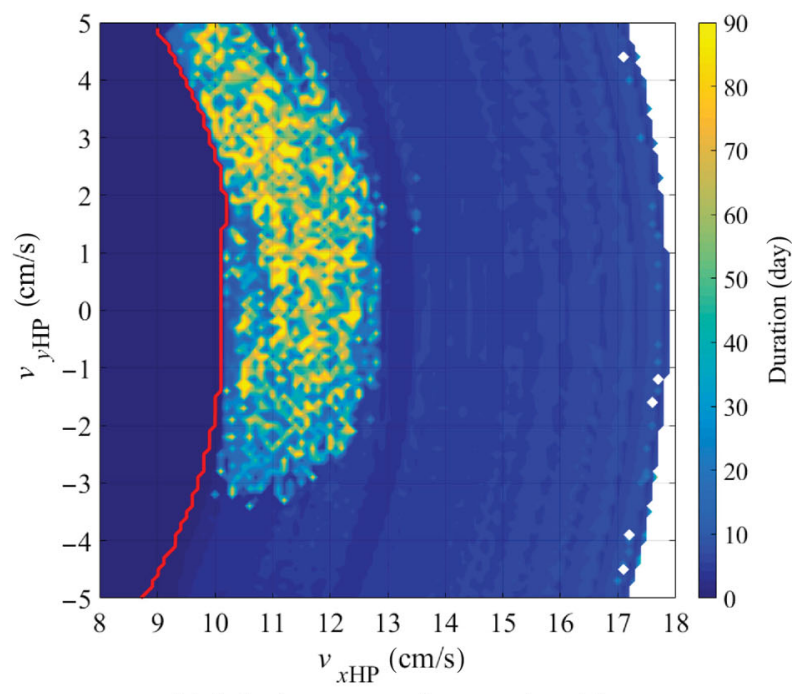

(b) Solution space of prograde orbits

Fig. 14 Close-up of duration map for the case $r=1.5 \mathrm{~km}$.
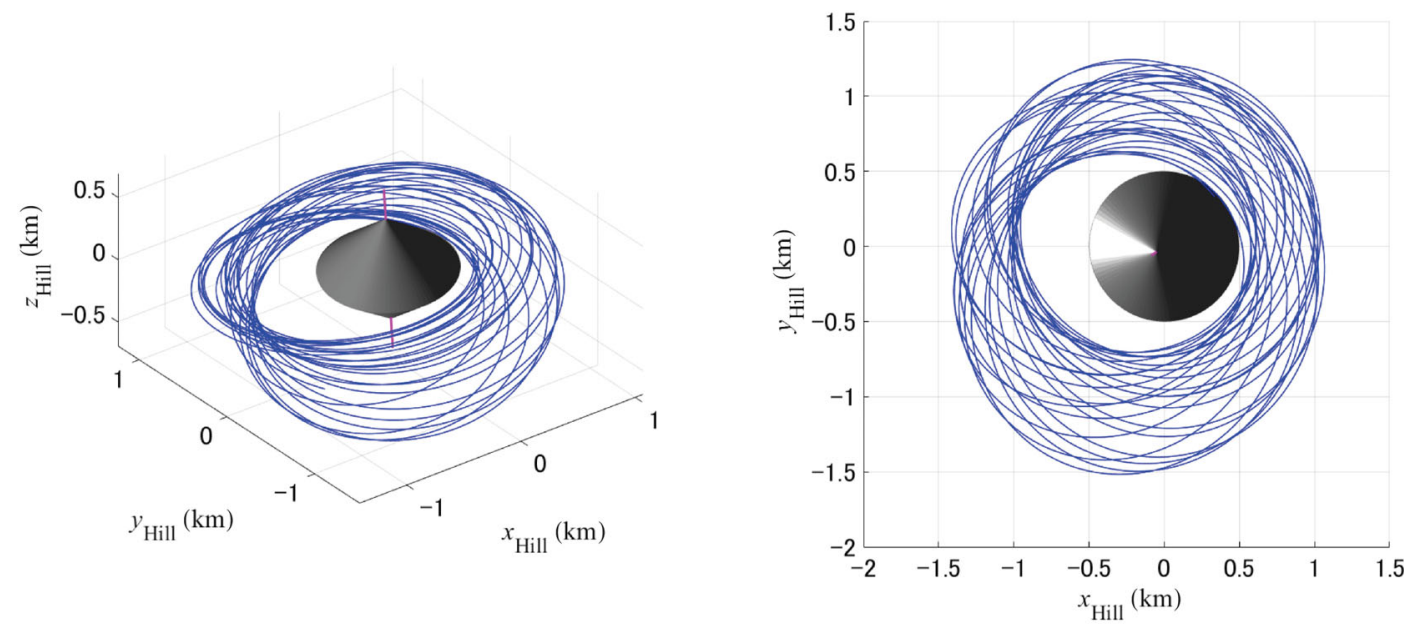

(a) Retrograde orbit $\left(\left[v_{x \mathrm{HP}}, v_{y \mathrm{HP}}\right]=[0.113,0] \mathrm{m} / \mathrm{s}\right)$
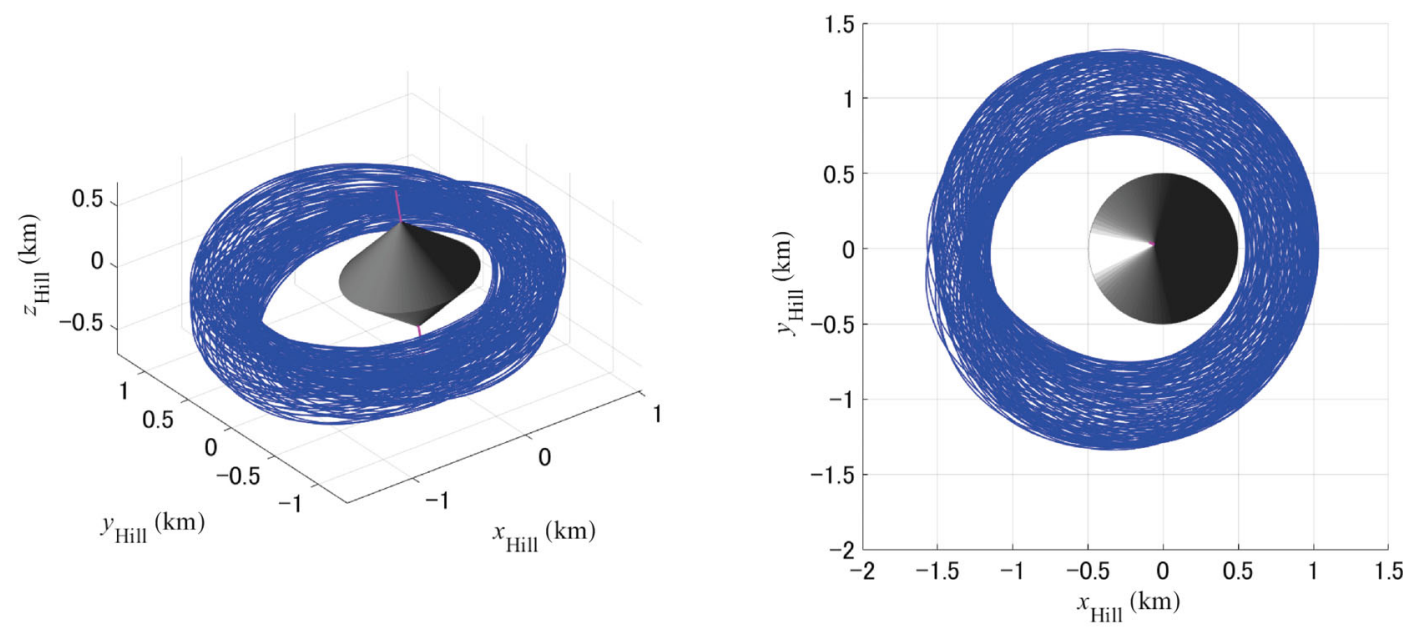

(b) Prograde orbit $\left(\left[v_{x \mathrm{HP}}, v_{y \mathrm{HP}}\right]=[-0.113,0] \mathrm{m} / \mathrm{s}\right)$

Fig. 15 Comparison of prograde orbit and retrograde orbit.

\section{(10) UNIIVRSITY PRESA}


precisely determine the initial $v_{x \mathrm{HP}}$ by considering various errors in using the spherical harmonics gravity model. Although the previous sections only consider the release velocity error, the actual orbit insertion has various associated errors, such as release angle error, reflectivity error, and spacecraft guidance and control error. Table 4 lists the insertion errors used in the Monte Carlo simulation. In addition to spacecraft and rover errors, dynamic uncertainty was also taken into account in the form of errors regarding higher order gravity. We conducted 500 Monte Carlo simulations for each initial condition. Figure 16 and Table 5 show the results. Figure 16 shows the orbits' periods ratio and duration ratio in four initial velocity cases $\left(v_{x \mathrm{HP}}=\right.$ $0.125,0.130,0.135,0.140 \mathrm{~m} / \mathrm{s})$. Significantly, the rover must avoid escape and an excessively long duration to the greatest possible extent. Table 5 lists the ratios of escape and long duration orbits. Although these results show there were no cases where both escape and long duration orbits were $0 \%$, we determined that $v_{x \mathrm{HP}}=0.13 \mathrm{~m} / \mathrm{s}$ was the best initial velocity because the escape ratio was less than $1 \%$ and the long duration ratio was also sufficiently low. Therefore, the case of $v_{x \mathrm{HP}}=0.13 \mathrm{~m} / \mathrm{s}$ was chosen as the nominal orbit and propagated in both the spherical harmonics and polyhedron gravity model, as shown in Fig. 17. Figure 18 shows each orbital element. The orbit duration of the spherical harmonics gravity model was 5.2180 days, whereas that of the polyhedron gravity model was
4.7933 days. Although the duration difference of about 0.4 days was caused by gravity and the differences in the surface model, the histories of the orbital element shown in Figs. 18(a) and 18(b) are approximately the same. Hence, propagation using the spherical harmonics gravity model was validated by the polyhedron gravity model.

Therefore, the nominal initial velocity was designed to be $\boldsymbol{v}_{\mathrm{HP}}=[0.13,0,0] \mathrm{m} / \mathrm{s}$. Since the rover was ejected in the $-y_{\mathrm{SC}}$ direction and the release velocity was not zero, the spacecraft gave both the orbital velocity of the rover and the compensation maneuver, where $\boldsymbol{v}_{\mathrm{HP}}=$ $[0.13,0.1534,0.0134] \mathrm{m} / \mathrm{s}$ when the rover was released.

\section{$5 \quad$ Flight results}

The MINERVA II-2 operation was performed between 28 September and 8 October 2019. Hayabusa2 started its descent along the sub-Earth line on 28 September. We repeated orbit determination and trajectory correction of the spacecraft daily so that the spacecraft arrived at the point where Rover 2 of MINERVA II-2 was deployed at 15:57:20 on 2 October. After releasing the rover, the spacecraft proceeded to the observation point, as shown in Fig. 19. The observation point was located at $\boldsymbol{r}_{\mathrm{HP}}=[2,0,8] \mathrm{km}$ in HP coordinate and the spacecraft aligned its attitude with the observation attitude. This is so the optical navigation camera (ONC-T) could image the area where

Table 4 Given errors in Monte Carlo simulation

\begin{tabular}{|c|c|c|}
\hline Error type & Value & Random type \\
\hline Rover 2 , release velocity error & from 5.4 to $25.4 \mathrm{~cm} / \mathrm{s}$ & Uniform random \\
\hline Rover 2, release angle error & $3 \sigma=5 \mathrm{deg}$ & Gaussian random \\
\hline Rover 2 , reflectivity error, $\gamma$ & from 0.2 to 0.5 & Uniform random \\
\hline Spacecraft guidance and control error, $x_{\mathrm{HP}}$ & $1 \sigma=80 \mathrm{~m}$ & Gaussian random \\
\hline Spacecraft guidance and control error, $y_{\mathrm{HP}}$ & $1 \sigma=80 \mathrm{~m}$ & Gaussian random \\
\hline Spacecraft guidance and control error, $z_{\mathrm{HP}}$ & $1 \sigma=20 \mathrm{~m}$ & Gaussian random \\
\hline Spacecraft guidance and control error, $v_{x \mathrm{HP}}$ & $1 \sigma=8.21 \mathrm{~mm} / \mathrm{s}$ & Gaussian random \\
\hline Spacecraft guidance and control error, $v_{y \mathrm{HP}}$ & $1 \sigma=8.19 \mathrm{~mm} / \mathrm{s}$ & Gaussian random \\
\hline Spacecraft guidance and control error, $v_{z \mathrm{HP}}$ & $1 \sigma=8.93 \mathrm{~mm} / \mathrm{s}$ & Gaussian random \\
\hline Gravity higher order, $C_{l m}(l \neq 0 \vee m \neq 0)$ & from $-3 \%$ to $3 \%$ & Uniform random \\
\hline
\end{tabular}

Table 5 Escape and long duration ratios

\begin{tabular}{ccc}
\hline$v_{x \mathrm{HP}}$ & Escape & Long duration (more than 40 days) \\
\hline $0.125 \mathrm{~m} / \mathrm{s}$ & $0 \%$ & $10.6 \%$ \\
$0.130 \mathrm{~m} / \mathrm{s}$ & $0.4 \%$ & $4.2 \%$ \\
$0.135 \mathrm{~m} / \mathrm{s}$ & $1.0 \%$ & $3.0 \%$ \\
$0.140 \mathrm{~m} / \mathrm{s}$ & $2.4 \%$ & $0.8 \%$ \\
\hline
\end{tabular}




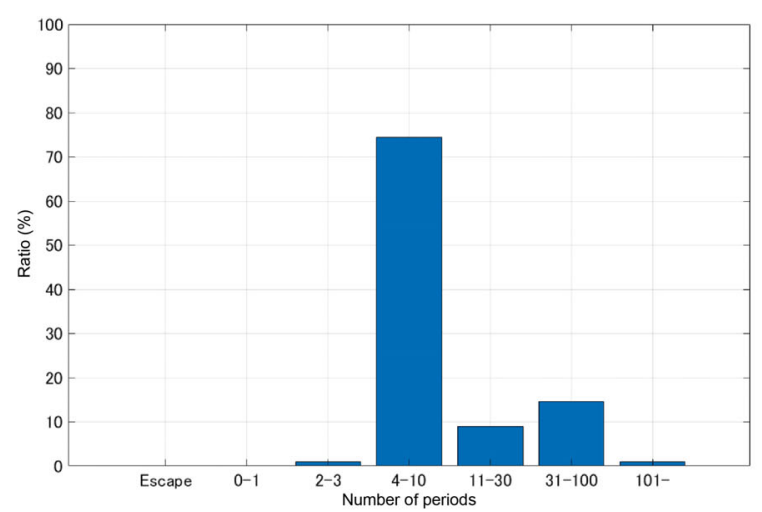

(a-1) Periods ( $\left.v_{x \mathrm{HP}}=0.125 \mathrm{~m} / \mathrm{s}\right)$

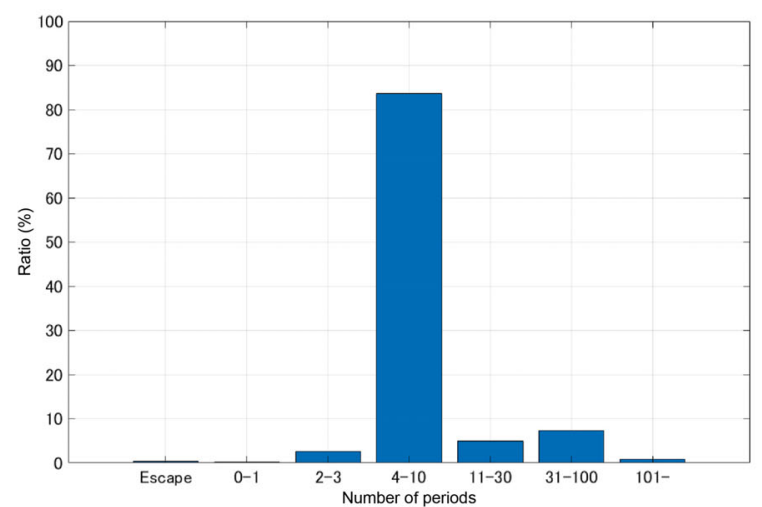

(b-1) Periods ( $\left.v_{x \mathrm{HP}}=0.13 \mathrm{~m} / \mathrm{s}\right)$

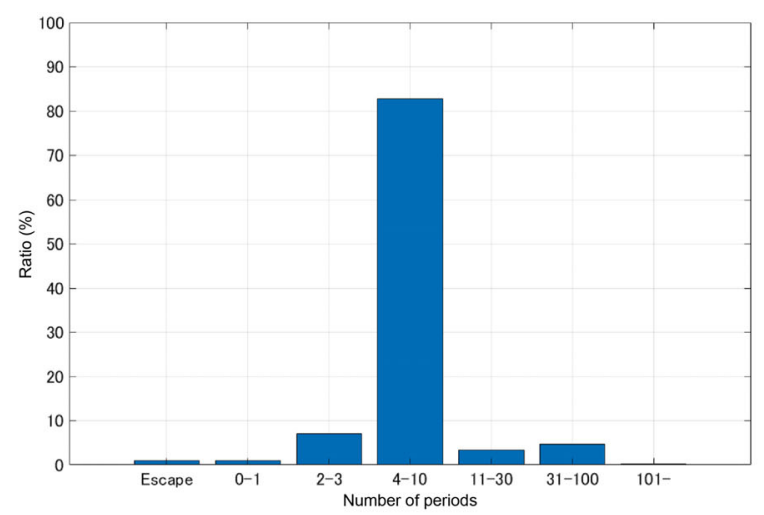

(c-1) Periods $\left(v_{x \mathrm{HP}}=0.135 \mathrm{~m} / \mathrm{s}\right)$

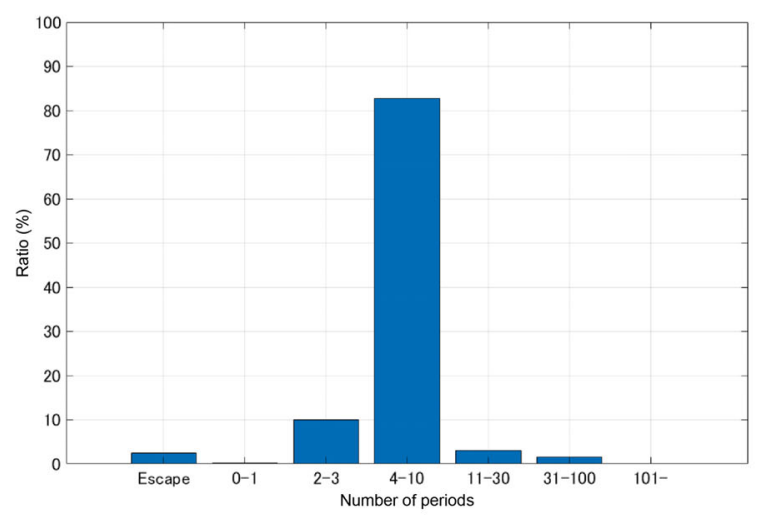

(d-1) Periods $\left(v_{x \mathrm{HP}}=0.14 \mathrm{~m} / \mathrm{s}\right)$

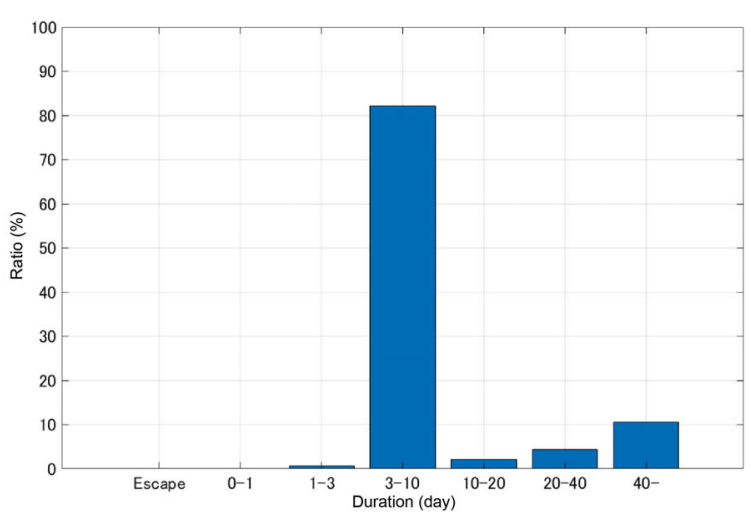

(a-2) Duration $\left(v_{x \mathrm{HP}}=0.125 \mathrm{~m} / \mathrm{s}\right)$

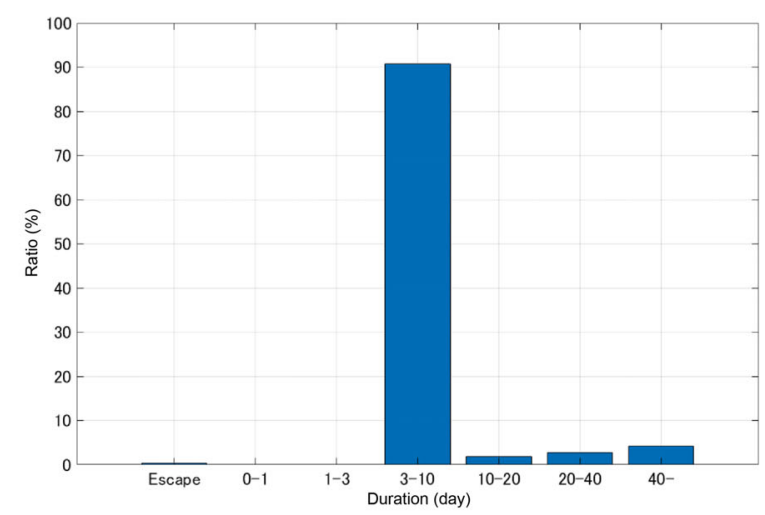

(b-2) Duration $\left(v_{x \mathrm{HP}}=0.13 \mathrm{~m} / \mathrm{s}\right)$

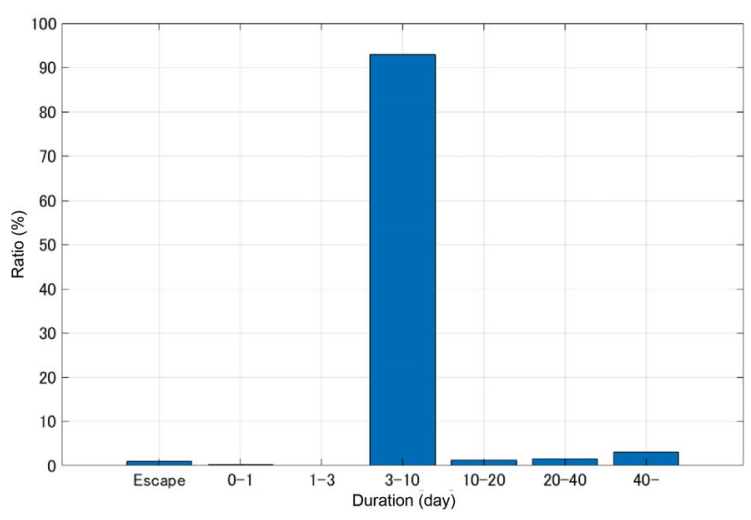

(c-2) Duration $\left(v_{x \mathrm{HP}}=0.135 \mathrm{~m} / \mathrm{s}\right)$

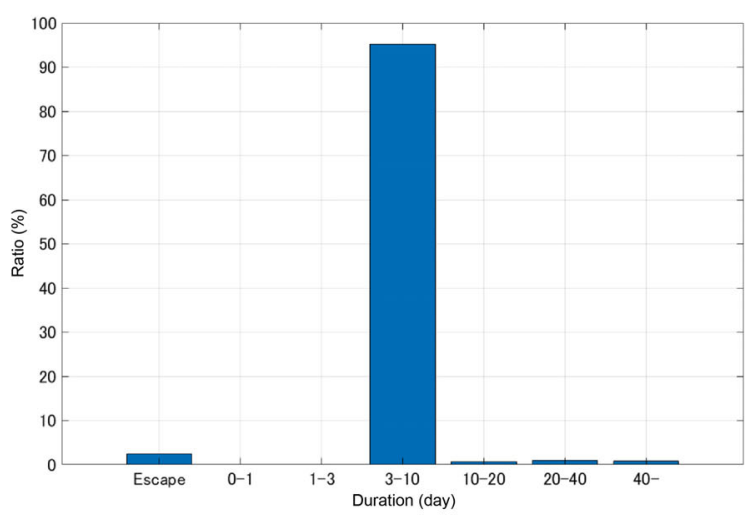

(d-2) Duration $\left(v_{x \mathrm{HP}}=0.14 \mathrm{~m} / \mathrm{s}\right)$

Fig. 16 Monte Carlo simulation results.

\section{(10) UNIVRSITY PRESA}



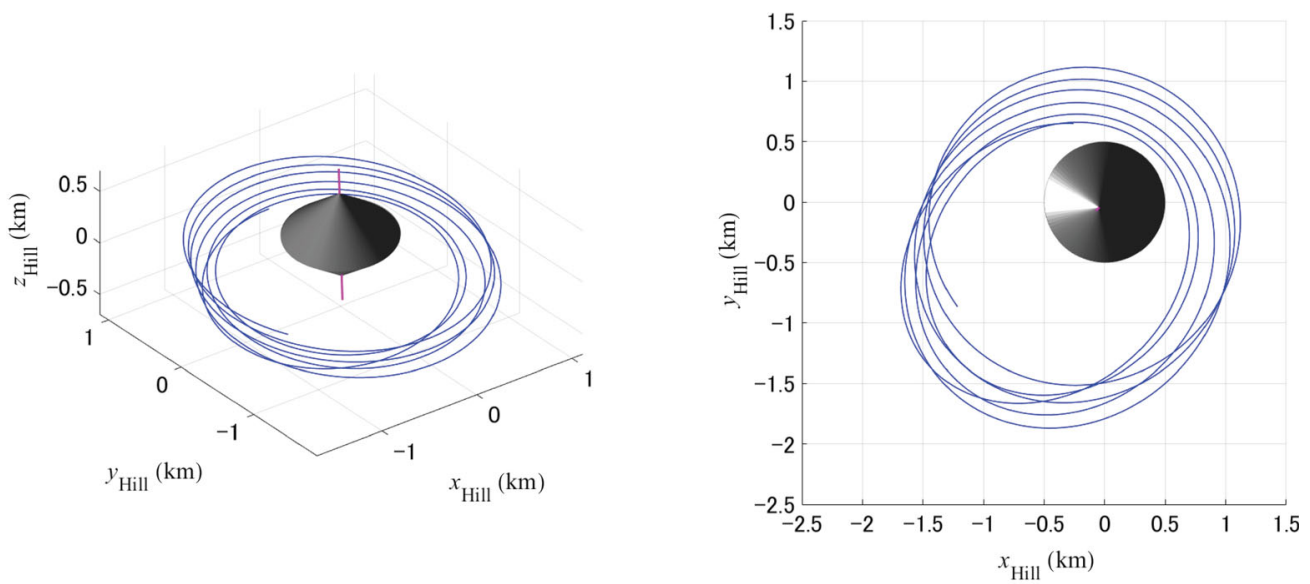

(a) Spherical harmonics gravity model
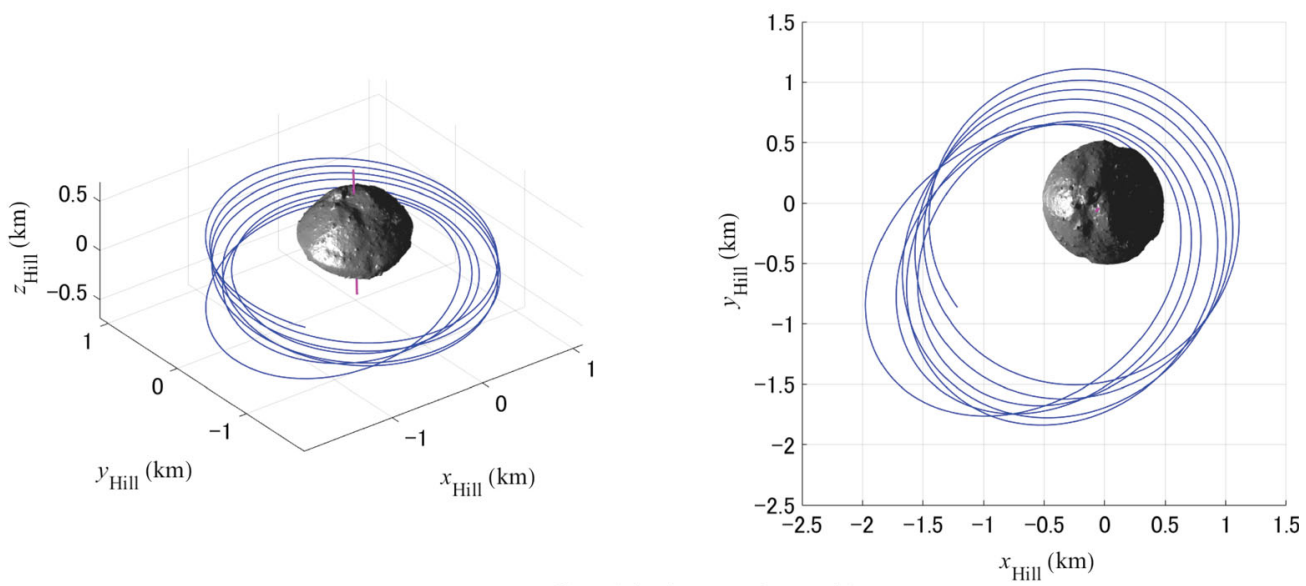

(b) Polyhedron gravity model

Fig. 17 Nominal orbit.
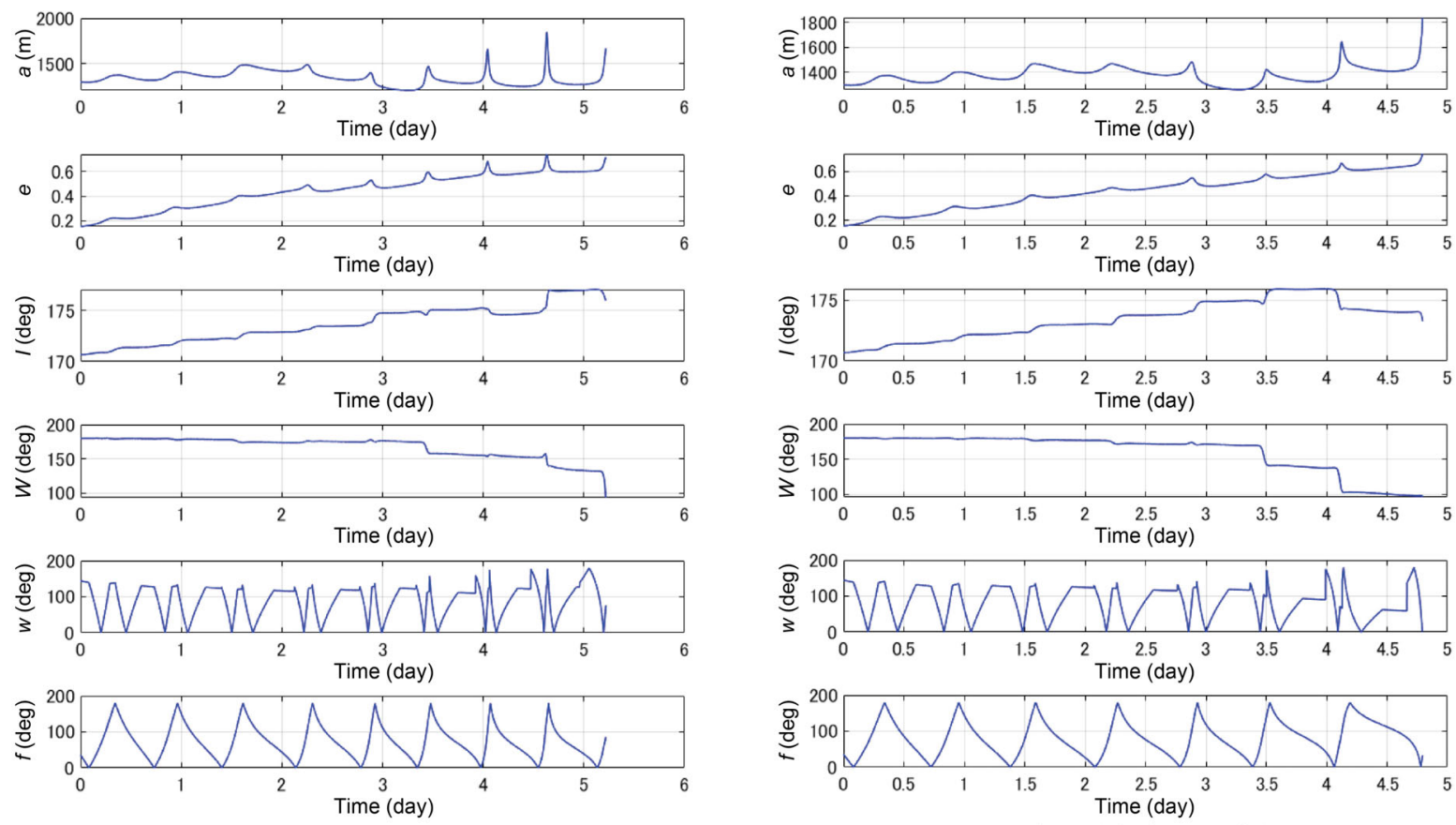

(a) Two-cone model

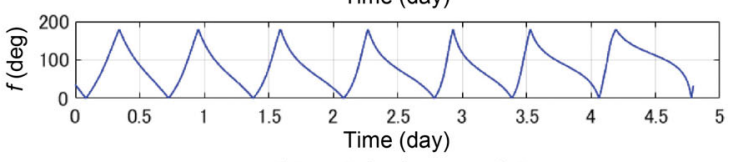

(b) Polyhedron model

Fig. 18 Nominal orbital element. 


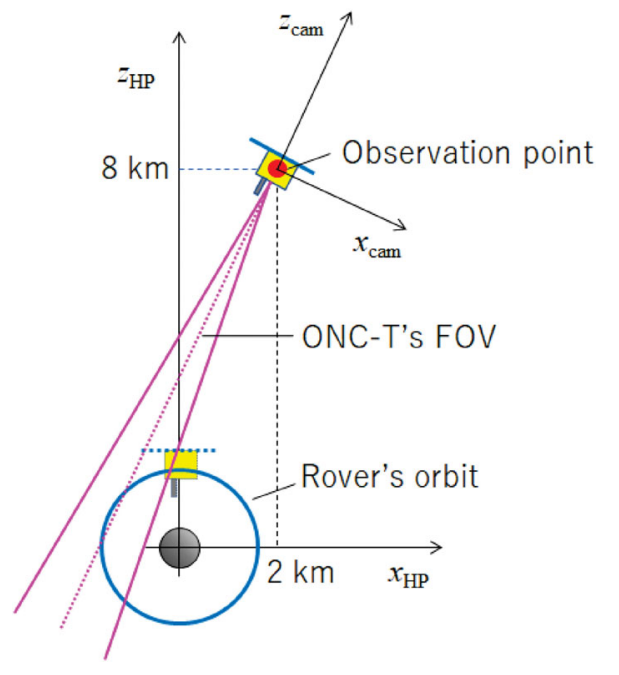

Fig. 19 Observation point and ONC-T coordinate $\boldsymbol{r}_{\text {cam }}$.

the rover was likely to pass, despite the release condition including the various errors mentioned above, and ONC$\mathrm{T}$ would not image Ryugu. Since ONC-T images of Ryugu were too bright, the rover could not be imaged if Ryugu was in the field of view (FOV) of ONC-T. After the spacecraft rotated $25 \mathrm{deg}$ around the $y_{\mathrm{sc}}$ axis, it rotated $-10 \mathrm{deg}$ around the $z_{\mathrm{sc}}$ axis, aligning its
HP attitude with the observation attitude. Here, the ONC-T coordinates are defined as the spacecraft fixed coordinates during the observation. The rover's position $\left(\boldsymbol{r}_{\text {cam }}\right)$ in ONC-T coordinates can be expressed as shown in Eq. (17).

$$
\begin{aligned}
\boldsymbol{r}_{\mathrm{cam}}= & \left(\begin{array}{ccc}
-1 & 0 & 0 \\
0 & 1 & 0 \\
0 & 0 & 1
\end{array}\right)\left(\begin{array}{ccc}
\cos \phi & \sin \phi & 0 \\
-\sin \phi & \cos \phi & 0 \\
0 & 0 & 1
\end{array}\right) . \\
& \left(\begin{array}{ccc}
\cos \theta & 0 & -\sin \theta \\
0 & 1 & 0 \\
\sin \theta & 0 & \cos \theta
\end{array}\right) \boldsymbol{r}_{\mathrm{HP}}
\end{aligned}
$$

where $\theta=25 \mathrm{deg}$ and $\phi=-10 \mathrm{deg}$. When the spacecraft fixed coordinates matched the HP coordinates, the $x$-axis of the ONC-T coordinates opposed that of the HP coordinates. After the spacecraft moved to the observation point, it continued to take pictures with ONC-T until 8 October 2019.

We obtained 14 pictures showing bright spots that indicate Rover 2 in orbit. Figure 20 shows the 14 pictures. These pictures were taken every $10 \mathrm{~min}$ from 08:20:17 to 10:23:15 on 3 October. We can see that the rover appears on the left side of the first picture and moves to the right side of the picture frame. Although
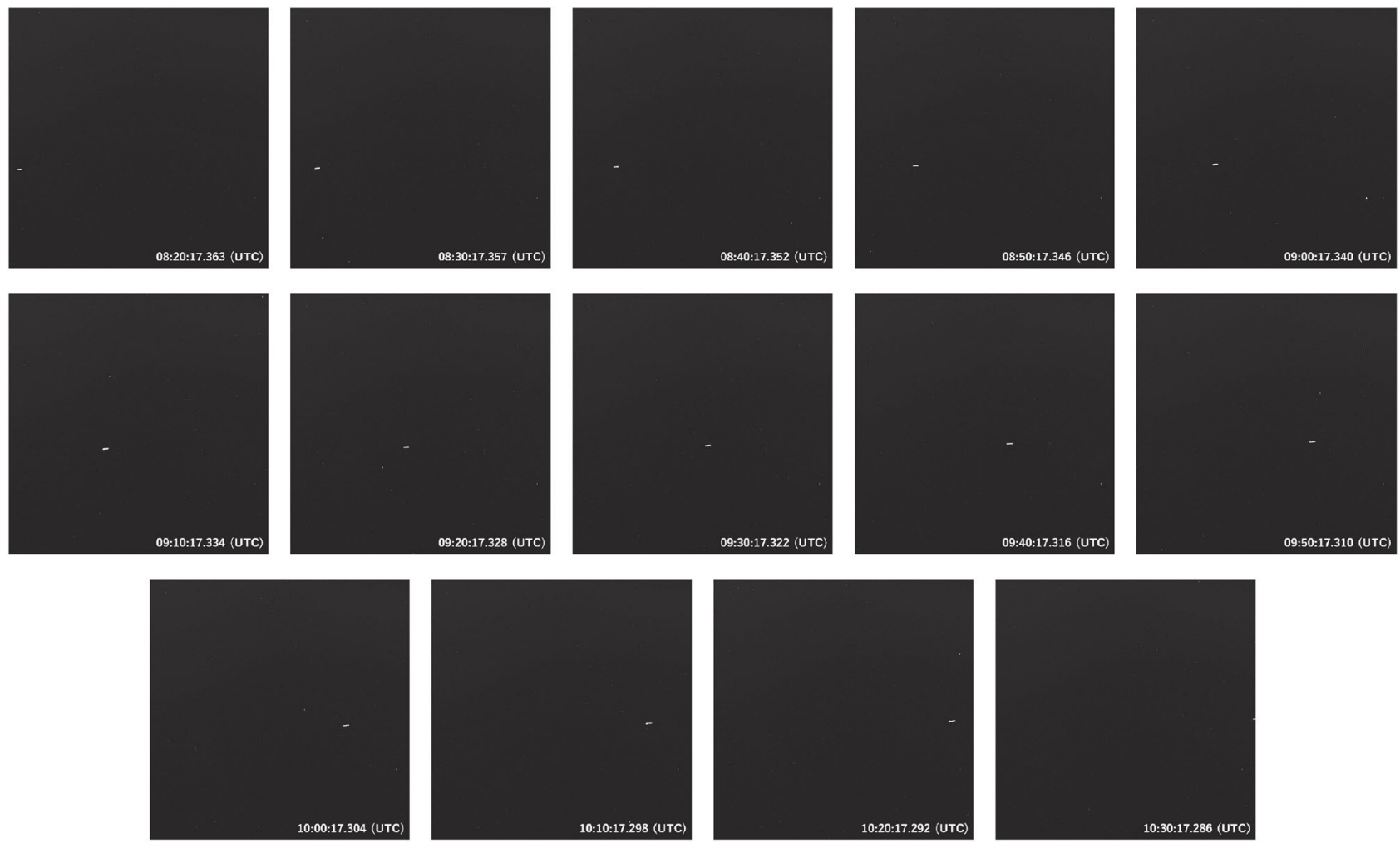

Fig. 20 Pictures taken by ONC-T.

(10) UNIINRSITY PUESA 
we planned to image the rover over several periods in case the rover was inserted into the nominal orbit, we were able to image one arc.

This study then approximately estimated the rover's release condition using these observation results. As a result of the orbit determination of Hayabusa2, when it descended we obtained the position of the rover's release as $\boldsymbol{r}_{\mathrm{HP}}=[11.56,-22.07,1503.78] \mathrm{m}$ at 15:57:20 on 2 October 2019 [12]. The rover's release velocity was estimated using least-squares estimation to minimize the objective function, as expressed below.

$$
J=\sum_{i}\left(x_{\mathrm{obs}, i}-x_{\mathrm{cal}, i}\right)^{2}+\left(y_{\mathrm{obs}, i}-y_{\mathrm{cal}, i}\right)^{2}
$$

where $x_{\mathrm{obs}, i}$ and $y_{\mathrm{obs}, i}$ denote the observed position of the rover in ONC-T coordinates, $x_{\mathrm{cal}, i}$ and $y_{\mathrm{cal}, i}$ denote the estimated position in ONC-T coordinates, and $i$ denotes the numbering of the pictures. The optimization problem was solved to minimize $J$ under the condition that the rover's initial velocity was set as the input parameters. This study did not estimate the gravity coefficients and fix them as nominal constant parameters. Table 6 lists the results of estimated initial velocity. Comparing the reference and estimated values, we found that the error in $v_{x \mathrm{HP}}$ was within $3 \sigma$ of the guidance and control error represented in Table 4. In addition, the release velocity magnitude, relative to the spacecraft, could be calculated as $0.1924 \mathrm{~m} / \mathrm{s}$ by $v_{y \mathrm{HP}}$ and $v_{z \mathrm{HP}}$ of the rover's estimated initial velocity and the spacecraft's compensation maneuver. The release velocity magnitude was also within the assumed error. However, the error of $v_{z \mathrm{HP}}$ was much larger than expected. Figure 21 shows the estimated position (calculation) and observed position (observation) in ONC-T coordinates. The O-C residual was found to converge and the estimated initial condition was valid. The estimated initial condition resulted in the orbit illustrated in HP coordinates, as shown in Fig. 22. The blue line indicates the rover's orbit, the green bold line indicates the arc taken by ONC-T, and the

Table 6 Comparison of the released rover's initial state

\begin{tabular}{ccc}
\hline & Reference & Estimation \\
\hline$x_{\mathrm{HP}}(\mathrm{m})$ & 0 & 11.56 \\
$y_{\mathrm{HP}}(\mathrm{m})$ & 0 & -22.07 \\
$z_{\mathrm{HP}}(\mathrm{m})$ & 1500 & 1503.78 \\
$v_{x \mathrm{HP}}(\mathrm{m} / \mathrm{s})$ & 0.13 & 0.1120 \\
$v_{y \mathrm{HP}}(\mathrm{m} / \mathrm{s})$ & 0 & -0.0128 \\
$v_{z \mathrm{HP}}(\mathrm{m} / \mathrm{s})$ & 0 & -0.0836 \\
\hline
\end{tabular}

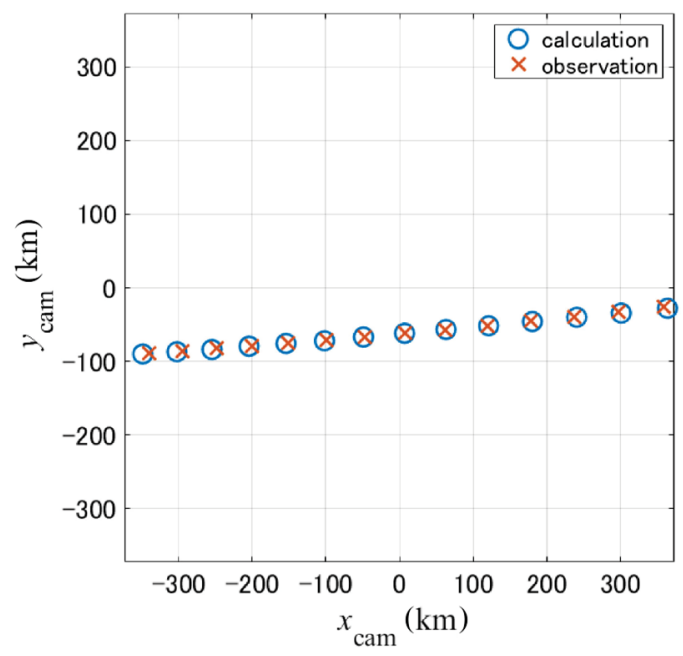

Fig. 21 Calculation and observation of MINERVA II-2's position in ONC-T's FOV.

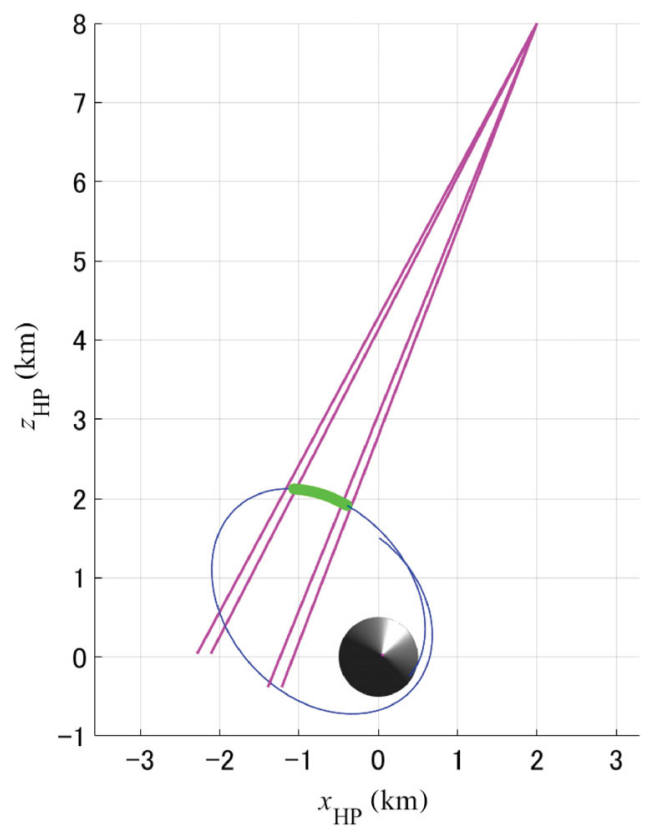

Fig. 22 Estimated MINERVA II-2 orbit with ONC-T's field of view.

four pink lines indicate the four corners of ONC-T's FOV. This figure indicates that after the rover orbited Ryugu for one and a quarter periods, it landed on the surface. The duration was 0.9372 days. The duration of all orbits in the Monte Carlo simulation, except for the cases of escape, were more than 3 days, as shown in Fig. 16(b-2). Nevertheless, the duration was much less than 3 days as the error in $v_{z \mathrm{HP}}$ was greater than expected. The actual release angle could be estimated based on the estimated initial velocity, as shown in Fig. 23. Although the angle between 


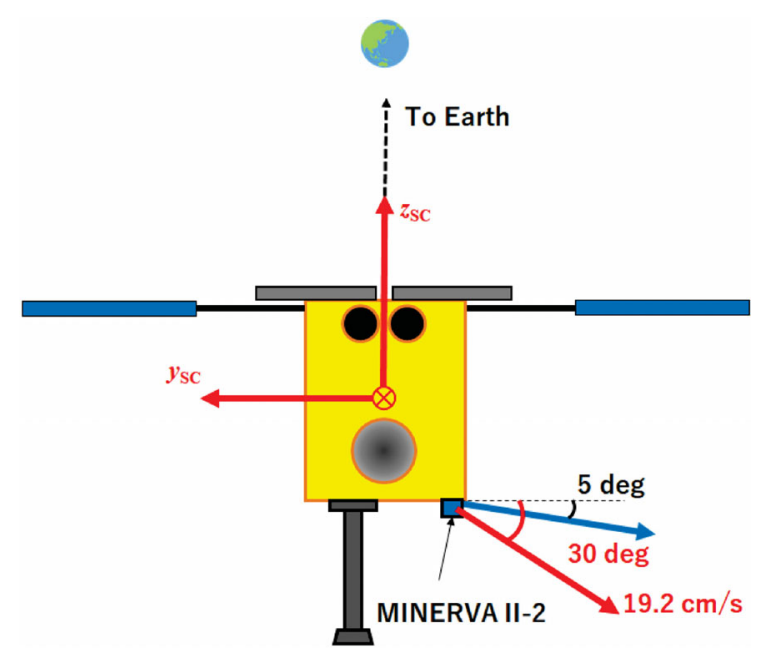

Fig. 23 Estimated MINERVA II-2's release angle.

the release direction and the $y_{\mathrm{SC}}$ axis was assumed to be $5 \mathrm{deg}$, it could be estimated as $30 \mathrm{deg}$. This corresponded with the analysis based on images taken by the other optical camera, in the seconds immediately following the rover's release. The MINERVA II-2 team concluded that the error in the release direction was attributed to inaccurate maintenance of the tightening torque of the screw used to close the rover's cover.

Thus, the MINERVA II-2 operation achieved orbiting of Ryugu and landing on the surface, although the periods were fewer and the duration shorter than planned. Even though SRP and gravitational irregularity strongly perturbed the dynamics around Ryugu and the rover had a large insertion velocity uncertainty, we successfully achieved Japan's first orbit around a small body for more than one period and established the possibility of a new method for gravity estimation.

\section{Conclusions}

This paper presented the design of the orbit of Ryugu by Rover 2 of MINERVA II-2, installed on the Hayabusa2 spacecraft. The rover required insertion into an orbit that would avoid escape from Ryugu and ultimately allow the rover to land on the surface. It was important to design a robust orbit satisfying these requirements under the severe conditions of the rover's large release uncertainty and the strongly perturbed dynamics around Ryugu by SRP and gravitational irregularity. Our analyses of the solution space revealed that the retrograde equatorial orbit was most appropriate as it satisfied operational safety and stability by taking advantage of the high obliquity of Ryugu. Moreover, the retrograde orbit offered a higher possibility of landing the rover than the prograde orbit. In addition, this study showed that Hayabusa2 mission accomplished not only orbiting of Ryugu but also landing on the surface via orbit determination, using the flight data of several images taken by ONC-T.

\section{Acknowledgements}

This work was partially supported by JSPS KAKENHI Grant (No. 18H01628).

\section{References}

[1] Nagaoka, K., Yoshida, K., Kurisu, M., Osuka, K., Tadakuma, K., Tsumaki, Y., Mineta, T., Kimura S., Narumi, T., Kubota, T., Yoshimitsu, T. Development of MINERVAII2, a micro-robot for asteroid surface exploration with innovative mobility. In: Proceedings of the 11th Low-Cost Planetary Missions Conference, 2015.

[2] Yoshimitsu, T., Kubota, T., Tomiki, A., Kuroda, Y., Development of hopping rovers for a new challenging asteroid. In: Proceedings of the 12th International Symposium on Artificial Intelligence, Robotics and Automation in Space, 2014: 5C-01.

[3] Soldini, S., Tsuda, Y. Assessing the hazard posed by Ryugu ejecta dynamics on Hayabusa2 spacecraft. In: Proceedings of the 26th International Symposium on Space Flight Dynamics, 2017.

[4] Scheeres, D. J., Marzari, F. Spacecraft dynamics in the vicinity of a comet. Journal of the Astronautical Sciences, 2002, 50(1): 35-52.

[5] Werner, R., Scheeres, D. Exterior gravitation of a polyhedron derived and compared with harmonic and mascon gravitation representations of asteroid 4769 Castalia. Celestial Mechanics and Dynamical Astronomy, 1997, 65(3): 313-344.

[6] Werner, R. A. Spherical harmonic coefficients for the potential of a constant-density polyhedron. Computers \& Geosciences, 1997, 23(10): 1071-1077.

[7] Kikuchi, S., Oki, Y., Saiki, T., Takei, Y., Takeuchi, H., Ono, G., Ikeda, H., Tsuda, Y. Retrograde teardrop orbits about asteroids: Application to the Hayabusa2 Mission. In: Proceedings of the 29th AAS/AIAA Space Flight Mechanics Meeting, 2019.

[8] Hesar, S. G., Scheeres, D. J., McMahon, J. W. Sensitivity analysis of the OSIRIS-REx terminator orbits to maneuver errors. Journal of Guidance, Control, and Dynamics, 2017, 40(1): 81-95. 
[9] Oki, Y., Tsuda, Y., Kawaguchi, J. Extension of stable terminator orbits around small bodies. Acta Astronautica, 2019, 157: 180-188.

[10] Lantukh, D., Russell, R. P., Broschart, S. Heliotropic orbits at oblate asteroids: Balancing solar radiation pressure and J2 perturbations. Celestial Mechanics and Dynamical Astronomy, 2015, 121(2): 171-190.

[11] Russell, R. P., Lantukh, D., Broschart, S. B. Heliotropic orbits with zonal gravity and shadow perturbations: Application at bennu. Journal of Guidance, Control, and Dynamics, 2016, 39(9): 1925-1933.

[12] Takeuchi, H., Yoshkawa, K., Takei, Y., Oki, Y., Kikuchi, S., Ikeda, H., Soldini, S., Ogawa, N., Mimasu, Y., Ono, G., et al. The deep-space multi-object orbit determination system and its application to Hayabusa2's asteroid proximity operations. Astrodynamics, 2020, 4(4): 377-392.

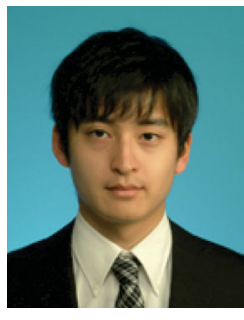

Yusuke Oki is a researcher at Japan Aerospace Exploration Agency (JAXA). He graduated with a master degree in astronautics from the University of Tokyo in 2016, and with a Ph.D. degree in astronautics from the University of Tokyo in 2019. He joined JAXA in 2019, and has been working on system design and orbit design of spacecrafts. His current research interests are astrodynamics, concurrent design, and deep space exploration. E-mail: oki.yusuke@jaxa.jp.

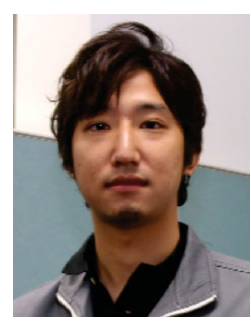

Kent Yoshikawa received his bachelor and master degrees in engineering from Tokyo Institute of Technology in 2013 and 2015, respectively. From 2015, he has been working as an engineer in Research and Development Directorate, JAXA. His current research interests include astrodynamics, GNC, planetary robotics, and planetary exploration. E-mail: yoshikawa.kento@jaxa.jp.

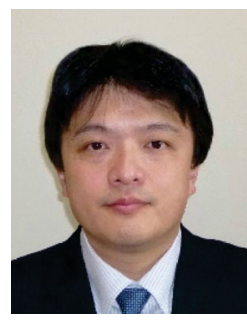

Hiroshi Takeuchi received his Ph.D. degree of science (physics and applied physics) from Waseda University in 2000. In 2006 he started working at Institute of Space and Astronautical Science (ISAS)/JAXA as a member of deep space orbit determination group. He was a visiting researcher of the NASA Jet Propulsion Laboratory in 2012-2013. He is currently an associate professor of ISAS/JAXA and is also the lead of the orbit determination of Hayabusa2. His current research interest is developing the deepspace multi-objects orbit determination system. E-mail: takeuchi@isas.jaxa.jp.

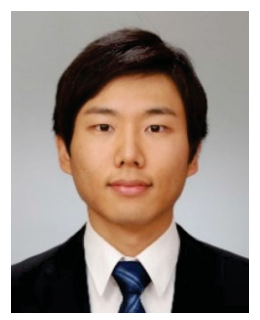

Shota Kikuchi received his Ph.D. degree in aeronautics and astronautics from the University of Tokyo in 2018. From 2015 to 2017, he served as a visiting scholar at Purdue University and NASA Jet Propulsion Laboratory. He is currently a postdoctoral research associate at JAXA and is engaged in the Hayabusa2 asteroid sample return mission as a system engineer. His primary research interests lie in the field of astrodynamics, particularly in the dynamics around small bodies. E-mail: kikuchi.shota@jaxa.jp.

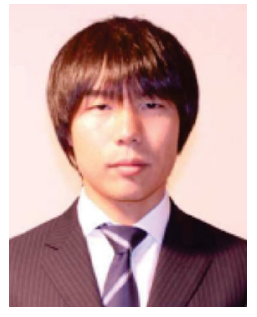

Hitoshi Ikeda received his Ph.D. degree from Kyusyu University, Japan, in 2008. $\mathrm{He}$ is a researcher at Research and Development Directorate, JAXA. He is involved in Hayabusa2 project as a radio science researcher. His research interests include astrodynamics, orbit determination, mission design, and flight dynamics operation. E-mail: ikeda.hitoshi@jaxa.jp.

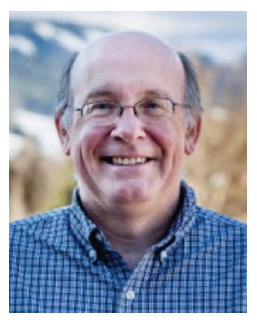

Daniel J. Scheeres is a distinguished professor in the Smead Department of Aerospace Engineering Sciences, University of Colorad. He holds degrees from the University of Michigan and Calvin College. Prof. Scheeres is a member of the National Academy of Engineering, a fellow of the American Institute of Aeronautics and Astronautics and of the American Astronautical Society.

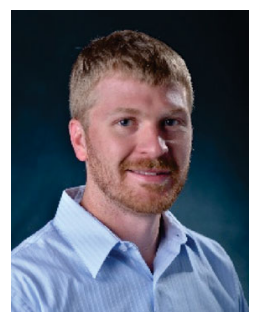

Jay McMahon is an assistant professor in the Smead Aerospace Engineering Sciences department at the University of Colorado Boulder, where he heads the ORCCA Laboratory. He earned his Ph.D. degree from the University of Colorado Boulder, an M.S. degree from the University of Southern California, and a B.S. degree from the University of Michigan. He is an associate fellow of the American Institute of Aeronautics and Astronautics. E-mail: jay.mcmahon@colorado.edu. 


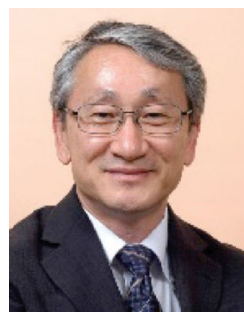

Junichiro Kawaguchi received his doctor degree of engineering from The University of Tokyo in 1983. He got the full professor position at ISAS in 2000 . He had been the Project Manager of the Hayabusa mission since 1996 till 2011. He is currently at senior fellow position, JAXA. He was the president of Japan Society for Space and Astronautical Science. And he also served Secretary General at the Strategic Headquarters for Space Policy's, Cabinet Secretariat, Government of Japan. He had been a full member and has been a corresponding member at the Science Council of Japan, Government of Japan. He has been a fellow at the Japan Society for Space and Astronautical Science. He has been a full member, board of Trustee at the International Academy of Astronautics. E-mail: kawaguchi.junichiro@jaxa.jp.

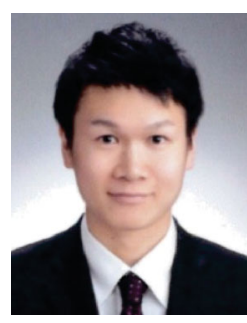

Yuto Takei received his Ph.D. degree in mechanical and aerospace engineering from Tokyo Institute of Technology, Japan, in 2015. He served as a visiting scholar at Institute of Robotics and Mechatronics of German Aerospace Center (DLR) in 2013. He is currently a researcher at Research and Development Directorate, JAXA. He is involved in Hayabusa2 project as a systems engineer. His research interests include astrodynamics, spacecraft system, space robotics, and deep space exploration. E-mail: takei.yuto@jaxa.jp.

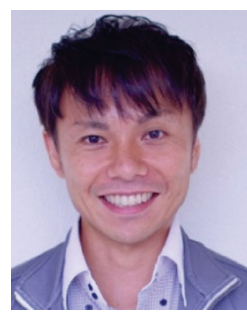

Yuya Mimasu is a researcher at JAXA. He graduated with a Ph.D. degree in aerospace engineering from Kyushu University. After graduation he joined JAXA, and has been working on guidance, navigation and control subsystem of Hayabusa2 mission which is JAXA's sample return mission from the asteroid. His research interests are astrodynamics and mission analysis around the small body. E-mail: mimasu.yuya@jaxa.jp.

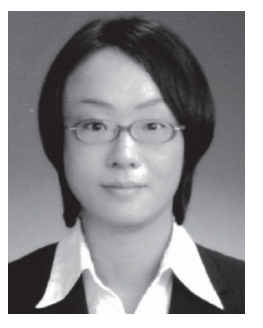

Naoko Ogawa received her B.E., M.E. and Ph.D. degrees in mathematical engineering and information physics in 2000, 2002, and 2005, respectively, from the University of Tokyo, Japan. From 2004 to 2008, she has been a research fellow of the Japan Society for the Promotion of Science. Since 2008 she has been a research engineer at ISAS, JAXA. Her current research interests include astrodynamics, mission design, robotics, spacecraft systems and operation. E-mail: naoko.ogawa@isas.jaxa.jp.

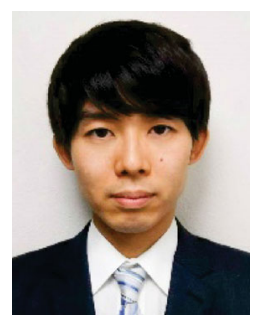

Go Ono is a researcher at JAXA. He graduated with a master degree of engineering from the University of Bath in 2011 and a Ph.D. degree in aerospace engineering from the University of Tokyo in 2014. He joined JAXA in 2015, and has been working on guidance, navigation, and control systems of JAXA's deep space missions such as Hayabusa2 and MMX. His current research interests are astrodynamics and deep space exploration. E-mail: ono.go@jaxa.jp.

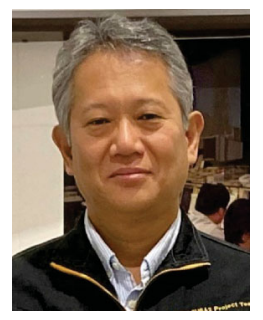

Fuyuto Terui received his Ph.D. degree in aerospace engineering from University of Osaka Prefecture in 1989. He has been a staff member of Space Technology Research Center of National Aerospace Laboratory (NAL) of Japan since 1989. He was a visiting scholar of University of Cambridge, Engineering Department, Control Group between 1994 and 1995. After the reorganization of space agencies in Japan, he has been a staff member of JAXA since 2003, and is now a function manager of "Hayabusa2" project as well as a representative of Attitude and Orbit Control System of "Hayabusa2" spacecraft. His main research field is robust control and image-based guidance, navigation, and control of a spacecraft such as debris removal space robot and the asteroid exploration probe. E-mail: terui.fuyuto@jaxa.jp.

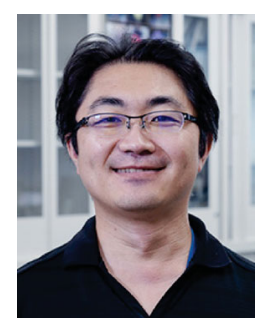

Manabu Yamada received his Ph.D. degree in Earth and planetary sciences from Hokkaido University, Japan, in 2006. He is a staff scientist at Planetary Exploration Research Center (PERC), Chiba Institute of Technology. He is now involved in Hayabusa2 project. He was a developer of the Optical Navigation Camera (ONC) system. His research interests include planetary atmospheres, remote sensing, and deep space exploration. E-mail: manabu@perc.it-chiba.ac.jp.

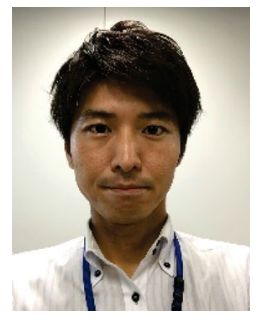

Toru Kouyama is a senior researcher at National Institute of Advanced Industrial Science and Technology (AIST). He graduated with a master degree in Earth and planetary science from the University of Tokyo in 2008, and with a Ph.D degree in Earth and planetary science from the University of Tokyo in 2012. He has entered AIST in 2012, and has been working on remote sensing research and satellite sensor calibration. His current research interests are planetary science, remote sensing, and deep space exploration. E-mail: t.kouyama@aist.go.jp. 


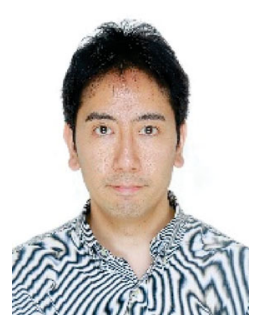

Shingo Kameda is a professor in Rikkyo University. He graduated with a master degree in science from the University of Tokyo in 2004, and with a Ph.D. degree in science from the University of Tokyo in 2007. He joined Rikkyo University in 2011 and has been working on development of scientific instruments onboard spacecraft for solar system exploration. His current research interest is planetary science. E-mail: kameda@rikkyo.ac.jp.

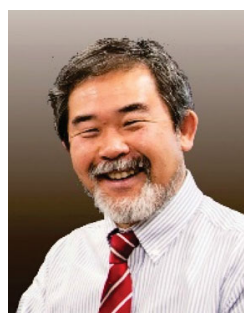

Kazuya Yoshida received his D.Eng. degree in mechanical engineering science from Tokyo Institute of Technology in 1990. Since 2003 he has been a full professor in Department of Aerospace Engineering, Tohoku University, Japan. His research field is space robotics and he has been contributing to space engineering education for international students at International Space University in Strasbourg, France.

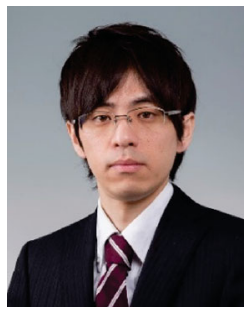

Kenji Nagaoka received his B.E. degree in aerospace engineering from the Tokyo Metropolitan Institute of Technology, Japan, in 2006. He also received his Ph.D. degree from the Graduate University for Advanced Studies (SOKENDAI), Japan, in 2011. From 2011 to 2019, he had been an assistant professor in the Department of Aerospace Engineering, Tohoku University, Japan. Since 2019, he has been an associate professor in the Department of Mechanical and Control Engineering, Kyushu Institute of Technology, Japan. His current research interests include space robotics, such as on-orbit servicing robots and planetary rovers. E-mail: nagaoka@ieee.org.

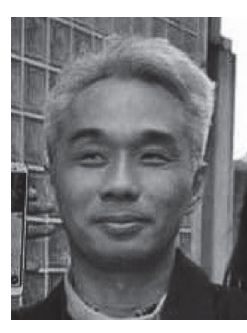

Tetsuo Yoshimitsu received his B.E., M.E., and D.E. degrees in electrical engineering from the University of Tokyo in 1992, 1994, and 2000, respectively. $\mathrm{He}$ is currently an associate professor at ISAS, JAXA. He was the principal investigator of MINERFVA payload in Hayabusa asteroid sample return mission. He has been the principal investigator of MINERVA-II rovers in Hayabusa2 mission, which achieved the world-first robotic mobile exploration on small planetary body. His research interests lie on robotic exploration system and control in space, such as planetary rovers, mobile system, path planning over natural terrains, localization on planetary surface and autonomous exploration system.

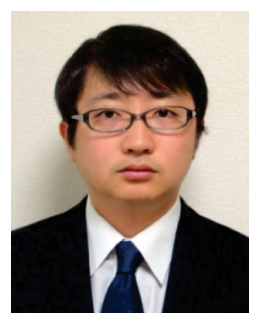

Takanao Saiki received his Ph.D. degree in aeronautics and astronautics from the University of Tokyo, Japan, in 2005. He is an assistant professor at ISAS, JAXA. He is now involved in Hayabusa2 project. He was a chief developer of the impact system and he is currently a project engineer. His research interests include astrodynamics, spacecraft system, and deep space exploration. E-mail: saiki.takanao@jaxa.jp.

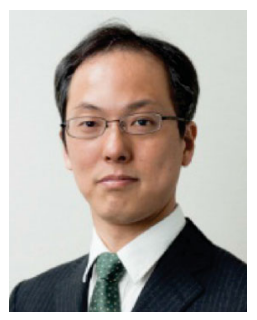

Yuichi Tsuda received his Ph.D. degree in aeronautics and astronautics from University of Tokyo in 2003, and joined JAXA in 2003 as a research associate. He was a visiting scholar of Department of Aerospace Engineering, University of Michigan and Department of Aerospace Engineering Sciences, University of Colorado Boulder in 2008-2009. He was a deputy lead of the IKAROS project, the world's first interplanetary solar sail mission. He is currently a professor of ISAS/JAXA and is also the project manager of the Hayabusa2, an asteroid sample-return mission. His research interests are astrodynamics, spacecraft system, and deep space exploration. E-mail: tsudayuichi@jaxa.jp.

Open Access This article is licensed under a Creative Commons Attribution 4.0 International License, which permits use, sharing, adaptation, distribution and reproduction in any medium or format, as long as you give appropriate credit to the original author(s) and the source, provide a link to the Creative Commons licence, and indicate if changes were made.

The images or other third party material in this article are included in the article's Creative Commons licence, unless indicated otherwise in a credit line to the material. If material is not included in the article's Creative Commons licence and your intended use is not permitted by statutory regulation or exceeds the permitted use, you will need to obtain permission directly from the copyright holder.

To view a copy of this licence, visit http://creativecommons.org/licenses/by/4.0/. 